\title{
Platform Flexibility Strategies: R\&D Investment versus Production Customization Tradeoff
}

\author{
Maud M. Van den Broeke ${ }^{1}$, Robert N. Boute ${ }^{2}$, and Jan A. Van Mieghem ${ }^{3}$ \\ ${ }^{1}$ IÉSEG School of Management, Lille, France \\ ${ }^{2}$ KU Leuven, Leuven, Belgium and Vlerick Business School, Gent, Belgium \\ ${ }^{3}$ Kellogg School of Management, Northwestern University, Evanston, Illinois, USA
}

March 19, 2018

\begin{abstract}
Product platforms are assets that are shared by multiple products. We study the optimal investment in platform flexibility. Each platform type is characterized by its functionality that determines its $R \& D$ investment and unit production cost, as well as the customization cost to produce the end products from the platform. The firm can invest in a portfolio of specialized platforms that align with the functionalities of a specific product and flexible platforms that cover the functionalities of a product range at lower customization cost. We characterize the optimal platform portfolio strategy using an ex-ante investment versus ex-post production customization tradeoff curve and show comparative statics of these costs, demand forecast, and the decision maker's regret and risk attitude. Flexible platforms provide operational hedging for risk-averse decision makers who thus should invest more than risk-neutral counterparts. In contrast to manufacturing flexibility, the regret of sub-optimal investments increases as demand is more negatively correlated.
\end{abstract}

Keywords: manufacturing; product development; platform; flexibility; newsvendor networks

\section{Introduction}

Product platforms are 'the collection of assets (i.e., components, processes, knowledge, ...) that are shared by a set of products' (Robertson and Ulrich, 1998, p. 20). These flexible assets facilitate cost-efficient product variety that is increasingly desired in markets characterized by more customer heterogeneity, fiercer competition, and fast evolving technology.

Much has been written on the benefits and disadvantages of product platforms in production and supply chain management (see e.g., Desai et al., 2001; Krishnan and Gupta, 2001; Simpson et al. 2014). Little research attention, however, has been devoted to a key decision in platformbased product development: how much investment in platform flexibility is desirable? Which type 
of platform, or which portfolio of flexible platforms should be developed, and how are these decisions impacted by demand and cost uncertainty and a manager's attitude towards risk?

Platform development often requires substantial R\&D investments: Volvo invested over USD 11 billion in its scalable platform architecture, which from 2016 onwards is used to derive all its different car models (Volvo, 2014). At the same time, evolutions in the automotive industry force firms to adapt their platform portfolio: In 2019, Volvo plans to bring its first all-electric vehicle to the market, based on a new modular electrification platform (Lambert, 2017). GM announced to reduce its number of platforms from 30 in 2010, down to four platforms by 2025 (Financial Times, 2012, 2015). These platform R\&D investment decisions are frozen far in advance of knowing actual product demand and can have large cost implications. In this article we present a stylized model to gain insight in the value-maximizing platform portfolio decision and its robustness, dependent on the demand forecast characteristics (demand scale, product scope, forecast uncertainty, and correlation between products), the cost structure of the fixed platform development versus the variable production and customization costs to end products, and the manager's regret and risk avoidance.

We develop a stochastic model with recourse of a two-product firm that offers a low-end and high-end product (product $j \in\{L, H\}$ ). In the first stage (ex-ante) the firm must decide which portfolio of platforms it should develop knowing only a product demand forecast. In the second stage (ex-post), the firm observes the actual demand $D=\left(D_{L}, D_{H}\right)$ and fulfills the demanded number of products by producing $D_{L}+D_{H}$ platforms and subsequently customizing them to the end products. The ex-ante investment in platform development is fixed in that it is independent of the later production quantities. In contrast, the ex-post production and customization costs are variable and incur a unit cost per unit produced.

We consider a platform portfolio that can include four types of platforms $i \in\{L, M, H, U\}$, respectively denoting a low-end, middle, high-end and ultimate platform. Each platform type $i$ is characterized by its functionality that determines the $\mathrm{R} \& \mathrm{D}$ investment cost $I_{i}$ and the unit production $\operatorname{cost} c_{i}$ to produce platform $i$, as well as the customization costs $c_{i j}$ to produce product $j$ from platform $i$, visualized in Figure 1, The low- and high-end platforms are specialized for respectively the low- and high-end products (therefore we will call them the two 'specialized' platforms). Developing only a low-end platform requires minimal investment and production costs, but it is under-designed for the high-end products and incurs high customization costs when it is upgraded to derive high-end products (Van den Broeke et al., 2015). Developing a high-end platform requires higher investment and production costs, but incurs lower customization costs when it is downgraded to derive low-end products. The middle platform functionality is 'in-between' the low-end and high-end products. Consequently, its investment, production, and customization costs are both somewhere between these two platform types. Finally, the ultimate platform is the most flexible according to the notion of flexible products by Alptekinoglu and Ramachandran (2015) and Chen et al. (2008) (where, for example, a firm can choose to offer a selection of specialized golf clubs or golf clubs with reconfigurable lofts that can be adapted by the consumer to her needs). The 


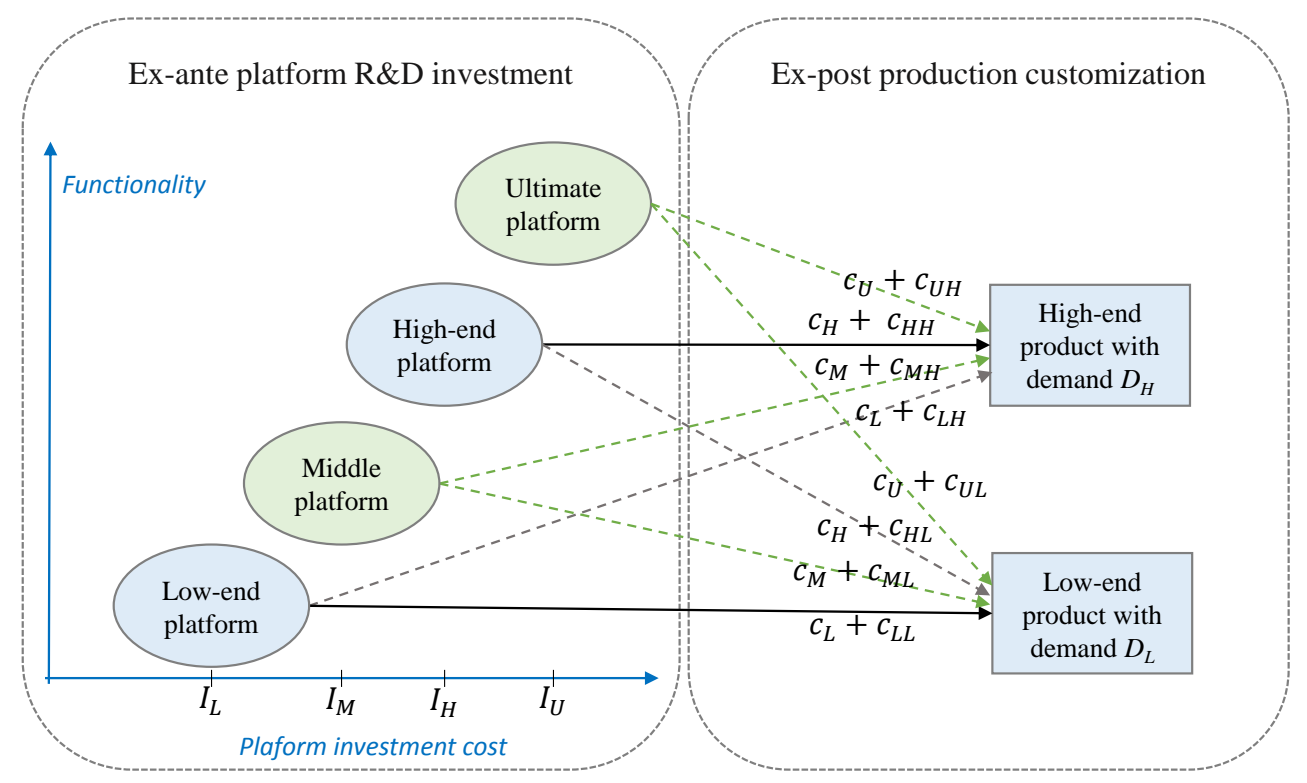

Figure 1: We model a firm that can develop a portfolio of four possible platforms $i \in\{L, M, H, U\}$ (left panel). Each platform can be customized to produce two vertically-differentiated products. Each platform has its own functionality and investment cost $I_{i}$, ex-post unit production cost $c_{i}$ and ex-post unit customization cost $c_{i j}$.

ultimate platform's ex-ante investment and ex-post production costs exceed the high-end platform costs, but its customization cost to derive either product is negligible, as the ultimate platform already contains the functionalities of each product. The ultimate platform is thus overdesigned for both the low- and high-end product. In contrast to the specialized low- and high-end platforms, the middle and ultimate platforms do not bet on one specific product. Therefore we will refer to them as 'flexible' platform investment strategies because they retain the flexibility to cost-efficiently deliver both the low-end and high-end product.

Our paper contributes in the following way:

1. We present a platform flexibility model and analytically characterize the optimal platform portfolio that minimizes total costs. Our analysis prescribes a firm's platform R\&D investment using an investment versus production and customization tradeoff curve. We show under which conditions it is optimal to develop a low-end or high-end platform (which we refer to as 'single specialized platform strategies'), a middle or ultimate platform (referred to as 'single flexible platform strategies'), or multiple platforms (i.e., a 'combined platform strategy').

2. We incorporate risk considerations by (i) evaluating regret, defined by the potential loss when making a sub-optimal platform investment, and (ii) risk, defined by the variance of ex-post production customization costs. We evaluate the value of perfect demand information and we identify the mean-variance frontier to incorporate the decision-maker's risk attitude. 
3. We show that investing in the development of a flexible (i.e., the middle or ultimate) platform can be optimal, even in the absence of demand uncertainty and a fortiori in the presence of risk aversion. Flexible platforms are effective operational hedges, meaning that they can reduce the variance of the future costs, while the expected costs increase only marginally. The latter effect is stronger in environments with higher demand uncertainty, higher developmentintensity of the platforms, and more negative correlation between product demands. Under negative correlation we find that the regret of developing a suboptimal platform portfolio increases. These insights are opposite to investments in manufacturing flexibility, such as flexible capacity investments.

Our research question spurs from a need at several companies to determine their optimal platform flexibility strategy. Barco, for instance, is a global technology company that introduced platforms in the design and production of its medical displays (Boute et al., 2018). The printed circuit board (PCB) was defined as the product platform. The different product variants are then obtained by adding electronic and mechanical components to the PCB. A key question is which platforms (PCBs) should be developed to serve their portfolio of products. For instance, the company has non-Fusion and Fusion displays, respectively seen as a low-end and high-end product, where the latter is capable of showing two images simultaneously. The required features (e.g., memory capacity, processor) of the PCB differ for both products. For Barco, it is relevant to know whether (or when) it is cost-beneficial to develop a specialized PCB with features that are aligned to one of the products, or a flexible PCB that can be customized at a lower cost to both products. Not only is future product demand unknown (beyond making a forecast) due to its dynamic technological market, the development of platforms is highly R\&D intensive and platforms serve around three to five years. The R\&D development costs include the time needed to develop a platform from scratch (including prototyping, certification and testing) by $R \& D$ engineers, and the design time needed to readjust the platform to new product variants. When a product is derived from one of the developed platforms, there are production costs for the PCB and customization costs which include the manufacturing costs of removing or soldering of components to the PCB, software additions, image calibration adjustments, etc.

Our model also provides insight into current industry practices. Consider the MQB platform that Volkswagen specialized for the Polo car (in our model seen as the low-end product) but also uses to derive the Audi A3 car (the high-end product) (Financial Times, 2013). This single specialized platform strategy of using one platform and deriving low-end products from their specialized platform and high-end products from an under-designed platform is only optimal in a restricted region of the demand space. Our model can assess under which demand scale and scope their current platform strategy is optimal, and which demand changes might trigger the use of a flexible platform.

Our problem setting and analysis is applicable to a range of similar investment decisions. For instance, a call center can train its employees for one specific task, which incurs a low investment in training but is (time-) expensive when they need to serve a different customer request. Alterna- 
tively, they can invest in training flexible employees that are more efficient to cope with a diverse set of customer requests. In summary, platform decisions capture the classic trade-off between specialization and flexibility.

\section{Literature Review}

Our work contributes to the literatures on platforms and on flexibility. Whereas Simpson et al. (2001) study the benefits of platforms to leverage high development costs over multiple products, Krishnan and Gupta (2001) and Van den Broeke et al. (2017) emphasize that these benefits also impose certain costs (e.g., due to over-design). This leads to a common platform only being appropriate under certain conditions. We adopt both benefits and costs to study the optimal platform portfolio strategy: how many platforms and how much platform flexibility should firms invest in? We further allow platforms to have higher quality than the low-end product, or even accommodate the performance levels of both the low- and high-end product. There is a vast body of literature on uncertainty and development flexibility reviewed by Krishnan and Ulrich (2001). Several studies consider obtaining flexibility in R\&D through managing the R\&D pipelines (e.g., by investing in parallel or sequential R\&D projects), given risk and uncertainty of the project and technology (Bhaskaran and Ramachandran, 2011; Childs and Triantis, 1999, Krishnan and Bhattacharya, 2002) and competitive intensity (Boonman et al., 2015, Kettunen et al., 2015). However, Zhang (2015) and Jiao et al. (2007) state that the literature on flexibility through the platform design is limited and primarily studies how physical components and functional attributes can be designed more flexibly from an engineering perspective to cope with market and technology changes; see also Li et al. (2013); Madni (2012); Suh et al. (2007).

The extensive literature on flexibility studies 'the ability to deal with changes and uncertainty' (Chod et al. 2010; Fine and Freund, 1990). Mix flexibility is the ability to cope with uncertainty on the product mix while volume flexibility refers to coping with uncertainty on the demand volumes or scale (Goyal and Netessine, 2011). Similarly, platform flexibility endows firms with the ability to leverage a platform investment over more volume or market size (economies of scale) and adapt to a diverse mix of products at a lower ex-post customization cost (economies of scope) (Chandler et al. 2009). Bassamboo et al. (2010) also consider investment in a flexible portfolio and distinguish between different levels of flexibility, where 'level- $k$ ' flexibility refers to the ability to process $k$ different product types. For instance, flexibility level $k=1$ refers to dedicated resources that can only offer one product, whereas $k=2$ captures resources that have the ability to process two different product types. In our model, both the low- and high-end product can be derived from all our platform types and thus all four platforms have flexibility level 2, but they differ in flexibility intensity: as the intensity of flexibility of the platform increases, its R\&D investment cost and production costs increase while its customization costs generally decrease.

Much of the flexibility research has focused on manufacturing flexibility — analyzing how much and what kind of capacity to invest in and how it should be distributed among customers as demand 
is realized (Bassamboo et al., 2010; Goyal and Netessine, 2011; Netessine et al., 2002, Van Mieghem, 1998; Yang and Ng, 2014) - and inventory substitution (Netessine et al., 2002, Shumsky and Zhang, 2009). Our study also involves downward and upward substitution, but platform design decisions do not involve the quantity restrictions that are essential in inventory or capacity allocation. Instead, platform decisions impact the variable customization costs to produce the end products. We find that the insights from manufacturing flexibility are not generalizable to platform investment decisions. Contrary to flexible capacity decisions where risk pooling is present, we find that the regret of sub-optimal platform decisions is higher under more negative correlation.

The impact of flexibility can be captured by option-based models (Huchzermeier and Loch, 2001). Our stochastic model with recourse and its analysis closely follows newsvendor network models and analysis (Van Mieghem, 1998) with the notable difference of binary investment decisions in uncapacitated assets. Indeed, the investment in platforms typically involves developing designs, processes and knowledge with ample capacity to derive products. Whereas newsvendor networks are originally designed for inventory management and capacity investment decisions, we adapt them to platform investments. Similar to the newsboy who must decide how many newspapers to buy before observing actual demand, a firm invests in platforms based on a demand forecast, and subsequently customizes these products once actual product demand is revealed. Newsvendor networks extend the classic newsvendor model by allowing for multiple products and different network configurations (in our case several platform combinations). The attraction of a newsvendor network analysis lies in the possibility of offering structural results that are amenable to analytic solution and graphical interpretation. Similar to newsvendor networks, we assume a linear cost structure where the expost production and customization costs are linear in production quantities. A difference, however, is that the ex-ante investments are binary variables: the decision is to either develop platform $i$ at $\operatorname{cost} I_{i}$ or not. We shall see that this results in scale and scope effects given that investment costs are fixed while production and customization costs vary with production quantities. To characterize the optimal combinatorial solution, we introduce an investment versus production customization tradeoff curve.

Insights on how demand uncertainty impacts platform decisions are missing in current literature (Krishnan and Gupta, 2001). Besides considering the average cost of a specific platform strategy, we also consider regret of making the wrong platform investment and the risk measured by the variability in costs. This approach is new in platform decisions, but has been implemented in capacity investment decisions (Van Mieghem, 2003) and decisions on the timing of investments (Chronopoulos et al., 2011). Risk-aversion can be insightfully captured through a mean-variance efficient frontier (Markowitz, 1991; Van Mieghem, 2007).

\section{Model}

We develop a stylized model with recourse of a firm that must decide on its platform portfolio to satisfy future demand for two products that differ in functionality. Denote the low-end product by 
$L$ and the high-end product by $H$. Let their demand over the platform lifetime be denoted by the vector $D=\left(D_{L}, D_{H}\right)$. Demand is assumed to be exogenous to the model.

Each product $j \in\{L, H\}$ can be developed from four types of platforms $i \in\{L, M, H, U\}$, where $L$ refers to a low-end platform, $M$ refers to a middle or mid-end platform, $H$ refers to a high-end platform and $U$ refers to an ultimate platform. Visualized in Fig. 1, developing a low-end and/or high-end platform are referred to as 'specialized' platforms because these platforms perfectly fit the (functionality) needs of respectively the low-end and high-end product. The middle and ultimate platform, referred to as 'flexible' platforms, are not perfectly specialized for either the low-end or high-end product, but can more easily (i.e., at a lower customization cost) be adapted to both products' (functionality) needs. Either one single platform can be developed or a portfolio of platforms.

The platform portfolio decision is denoted by the binary vector $Z=\left(x_{L}, x_{M}, x_{H}, x_{U}\right)$ where $x_{i}=1$ if the firm invests in platform $i \in\{L, M, H, U\}$ and zero otherwise. Higher platform functionality comes at a higher ex-ante $\mathrm{R} \& \mathrm{D}$ investment cost to develop platform $i$, denoted by $I_{i}$, with $I_{U}>I_{H}>I_{M}>I_{L}>0$. Denote the investment cost of platform portfolio $Z$ as

$$
I(Z)=Z \cdot I=\sum_{i \in\{L, M, H, U\}} x_{i} I_{i}
$$

Ex-post, once product demand $D_{j}$ is known, each time a platform $i$ is used to produce a product, a per unit production cost $c_{i}$ is incurred, with $c_{U}>c_{H}>c_{M}>c_{L}>0$. Each platform type $i$ can be used to produce either product $j$ at a customization cost $c_{i j} \geq 0$ per unit produced. Lower functionality platforms are 'under designed' and incur lower production costs, but higher customization costs to produce the high-end product than higher functionality platforms. The latter, at their turn, are 'over designed' to produce the low-end product. Without loss of generality, we assume there is no customization cost when deriving a product from its specialized platform:

$$
0=c_{L L}=c_{H H}<c_{H L}<c_{L H},
$$

where the latter assumes that deriving a product from an over designed platform is typically easier than from an under designed platform (for instance, at Barco de-activating functionalities (e.g., excluding an image quality-improving sensor) is cheaper than adding functionalities (e.g., by soldering extra components to the printed circuit board)). Likewise, we will also assume $c_{M L}<$ $c_{M H}$.

A middle platform is more flexible to customize to either a low-end and high-end product, as the functionality 'gap' between the platform and the product is smaller. Hence, we assume $c_{M L}<c_{H L}$ and $c_{M H}<c_{L H}$. While the ultimate platform is most expensive in investment and production, it provides the highest flexibility (i.e., lowest customization cost) ex-post. Summarizing the customization assumptions for the middle and ultimate platform:

$$
0 \leq c_{U L}<c_{M L}<c_{H L} \text { and } 0 \leq c_{U H}<c_{M H}<c_{L H}
$$


Note that the specialized platforms $L$ and $H$ can only be optimal if it is less costly to derive a product from its specialized platform than from an under- or overdesigned platform:

$$
c_{L}<c_{i}+c_{i L} \text { for } i \in\{M, H, U\} \quad \text { and } \quad c_{H}<c_{i}+c_{i H} \text { for } i \in\{L, M, U\} \text {. }
$$

Moreover, investing in a flexible platform $U$ can only be optimal if total ex-post costs are lower than when using another non-specialized platform:

$$
c_{U}+c_{U L}<c_{i}+c_{i L} \text { for } i \in\{M, H\} \quad \text { and } \quad c_{U}+c_{U H}<c_{i}+c_{i H} \text { for } i \in\{L, M\} .
$$

Using a middle platform $M$ to derive product $H$ can only be optimal if total ex-post costs are lower than when using platform $L$ :

$$
c_{M}+c_{M H}<c_{L}+c_{L H} .
$$

We assume that once the firm has invested in a portfolio $Z$ each platform has ample capacity to meet all product demand. For a platform portfolio $Z$ that only invests in one platform $i$ - i.e., $Z$ equals the $i$-th unit vector $e_{i}$-total ex-post variable costs $C(D, Z)$ are then simply

$$
C\left(D, Z=e_{i}\right)=c_{i L} D_{L}+c_{i H} D_{H}+c_{i}\left(D_{L}+D_{H}\right)
$$

When the platform portfolio includes multiple platforms, ex-post variable costs are minimized by deriving each product from the platform that is closest in functionality, i.e., the platform with the lowest sum of production and customization costs:

$$
C(D, Z)=c_{L}(Z) D_{L}+c_{H}(Z) D_{H}, \text { where } c_{j}(Z)=\min _{i: x_{i}=1} c_{i j}+c_{i} .
$$

Platform development typically requires substantial amounts of time and we assume that timeto-market is sufficiently important that the firm must make the platform decisions before actual demand is known (ex-ante). At Barco, the average platform development time takes around three years. Given its rapidly evolving market, postponing platform development until product demand is known is not an option. Thus investment decisions are based on a demand forecast, which we model by the multivariate probability distribution of $D$, while the production and customization decision can be postponed until actual demand is observed (ex-post) ${ }^{1}$ In a first phase we will consider a riskneutral decision maker that seeks to minimize expected costs. With exogenous demand, revenues are independent of the platform and customization decisions. Profit maximization thus reduces to the following cost minimization decision problem: Find a platform portfolio $Z^{*}$ that minimizes the total cost which is the sum of R\&D investment and expected production customization costs:

\footnotetext{
${ }^{1}$ We acknowledge a simplification inherent in our two-stage model: $D_{j}$ is demand over the lifetime of platforms, but knowing that demand accurately at the start of product life can be difficult for long product lives and would thus retain some uncertainty in practice.
} 


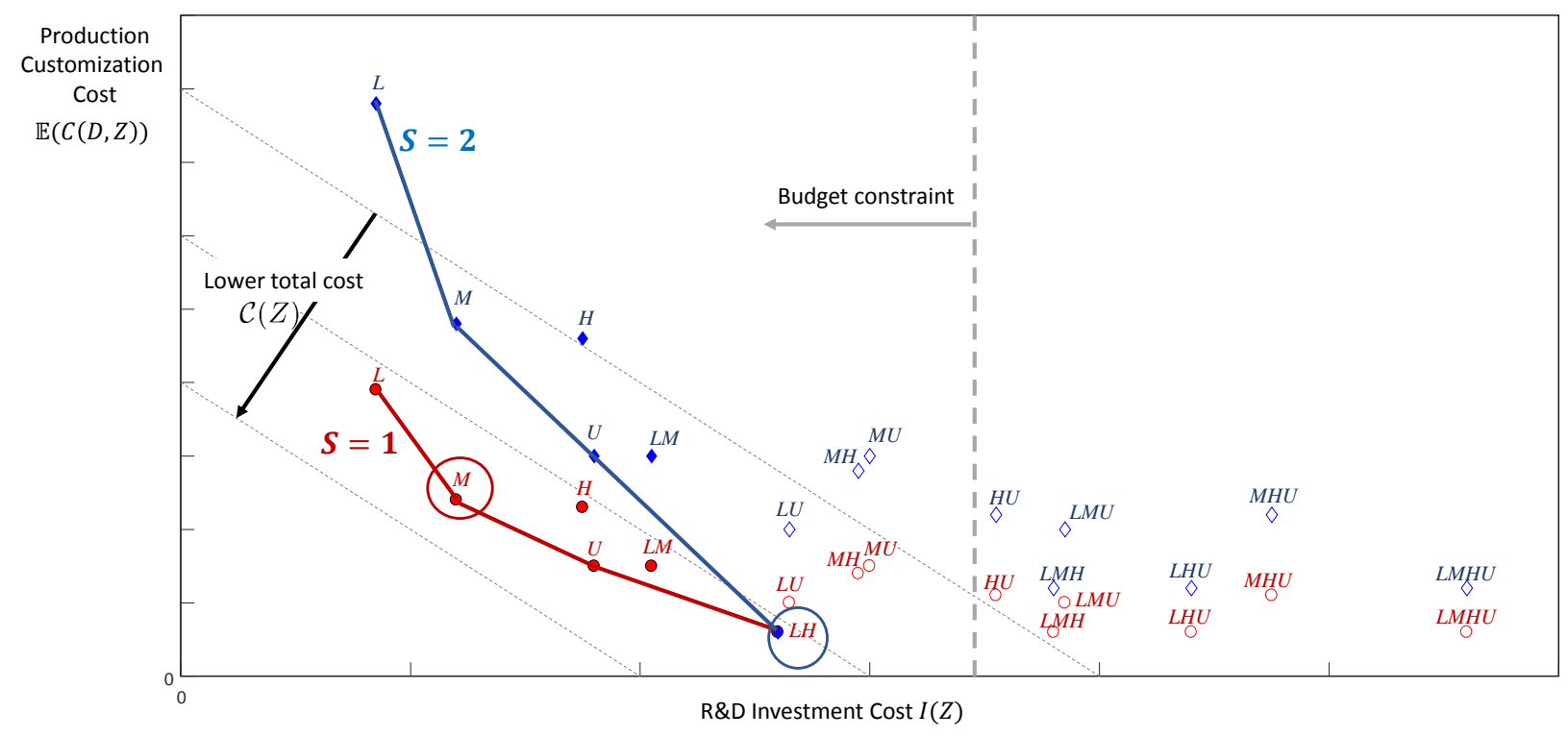

Figure 2: There are $2^{4}-1=15$ possible investment strategies $Z$ and corresponding points $(I(Z), \mathbb{E}(C(D, Z))$ in the "ex-ante investment versus ex-post production customization" cost space. The strategies on the trade-off curve (south-west part of the convex hull) dominate other strategies. The blue diamonds describe an environment where total expected product demand $\left(S=\mu_{L}+\mu_{H}\right)$ is twice as much as the one described by the red circles. Optimal strategies are circled and touch the lowest total cost isoline if they are budget feasible. As demand scales up, the same strategies remain on the trade-off curve but the optimal strategy can move 'down' the trade-off curve (e.g., $M$ to $L H)$. Note that, depending on the cost environment, the platform strategies on the trade-off curves can change.

$$
Z^{*}=\arg \min _{Z} \mathcal{C}(Z) \text { where } \mathcal{C}(Z)=I(Z)+\mathbb{E}(C(D, Z))
$$

\section{Optimal strategies and Investment versus Production Customiza- tion Trade-off Curves}

It is insightful to represent a platform strategy $Z$ by its corresponding point $(I(Z), \mathbb{E}(C(D, Z))$ in the "investment versus production customization" cost space. Given four binary platform type decisions $x_{i}$, there are $2^{4}-1=15$ possible investment strategies $Z$, consisting of a combination of investing in one or more platforms, and thus 15 points in the cost space. Figure 2 represents the portfolio problem visually.

The tradeoff curve is the "south-west" part of the convex hull of the 15 points, and thus is convex by construction. By definition, platform strategies on the tradeoff curve dominate other strategies. The optimal strategy touches the lowest isoline of total cost, which are the downward sloping lines with slope -1 in Figure 2. (It is possible to have multiple optimal strategies if the 
frontier has segments with slope -1). We can show that 9 platform strategies are always dominated (proof is relegated to the Appendix) so that we can write the set of optimal platform strategies as follows (slightly abusing notation):

Proposition 1. $Z^{*} \in \mathcal{Z}=\{L, M, H, U, L H, L M\}$. At most 6 platform strategies can ever be on the investment versus production customization tradeoff curve: only the four single-platform strategies $L, H, M, U$ and two combined platform strategies $L H$ and $L M$ can be optimal.

According to Proposition 1 it is never optimal to:

- develop more platforms than products;

- develop the ultimate platform in combination with other platforms;

- develop a high-end platform in combination with a middle platform.

Note that budget constraints can limit companies in their design variety during the ex-ante platform investment and development phase. This is straightforwardly incorporated in our model by adding the following (ex-ante) budget constraint $B$ to optimization problem (9):

$$
I(Z) \leq B
$$

As the budget $B$ reduces, the vertical budget constraint line in Figure 2 moves left. Eventually the budget starts restricting the trade-off curve, more platform combinations become budgetinfeasible (i.e., if $\sum_{i} x_{i} I_{i}>B$ ) and, consequently, the optimal strategy can change. Typically, as the budget no longer allows the development of two platforms, the flexible platforms become optimal for a larger demand space.

Denoting the forecasted mean demand vector by $\mathbb{E} D=\mu$, we directly take demand expectations. This yields the following total cost for each of the 6 possibly-optimal strategies $Z \in \mathcal{Z}$ :

$$
\mathcal{C}(Z)=I(Z)+\mathbb{E}(C(D, Z))= \begin{cases}I_{L}+c_{L H} \mu_{H}+c_{L}\left(\mu_{L}+\mu_{H}\right) & \text { if } Z=L, \\ I_{M}+c_{M L} \mu_{L}+c_{M H} \mu_{H}+c_{M}\left(\mu_{L}+\mu_{H}\right) & \text { if } Z=M, \\ I_{H}+c_{H} \mu_{L}+c_{H}\left(\mu_{L}+\mu_{H}\right) & \text { if } Z=H, \\ I_{U}+c_{U L} \mu_{L}+c_{U H} \mu_{H}+c_{U}\left(\mu_{L}+\mu_{H}\right) & \text { if } Z=U, \\ I_{L}+I_{M}+c_{M H} \mu_{H}+c_{L} \mu_{L}+c_{M} \mu_{H} & \text { if } Z=L M, \\ I_{L}+I_{H}+c_{L} \mu_{L}+c_{H} \mu_{H} & \text { if } Z=L H .\end{cases}
$$

It directly follows that the platform portfolio decision trades off investment cost with production customization costs evaluated at expected demand. The structure of these costs also directly allows the following comparative statics on the tradeoff curve. To express those, it is helpful to transform 


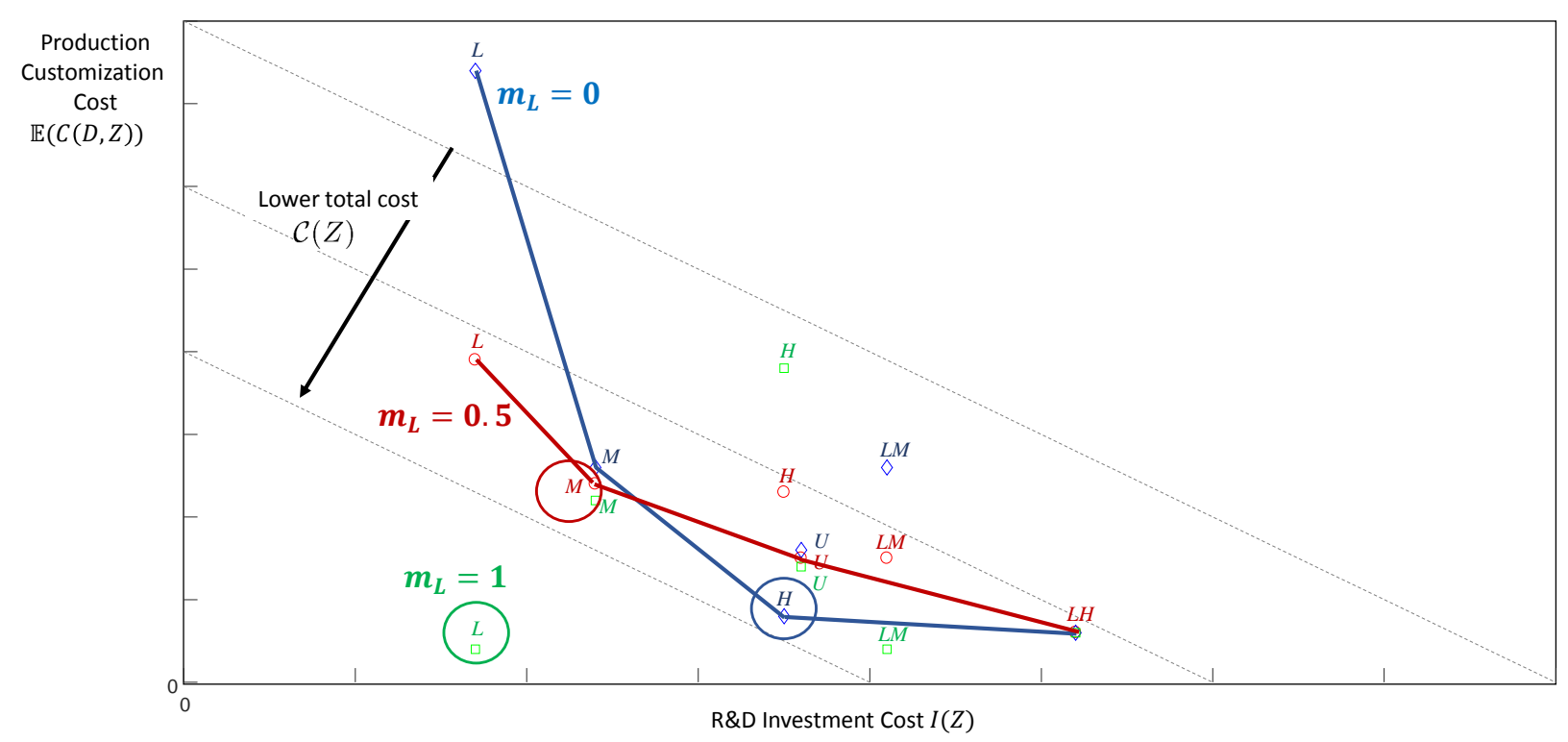

Figure 3: The trade-off curve moves up and to the right as the mix changes from the low product (green squares, $m_{L}=1$ ) to equal mix (red circles, $m_{L}=m_{H}=0.5$ ) to the high product (blue diamonds, $\left.m_{L}=1-m_{H}=0\right)$. Notice that the specialized platform strategies $L$ and $H$ are optimal at the extreme mix points whereas the flexible platforms gain in importance otherwise. Note that, depending on the cost environment, the platform strategies on the trade-off curves can change.

the expected demand vector in terms of total market size or scale $S=\mu_{L}+\mu_{H}$ and non-negative mix vector $\left(m_{L}, m_{H}\right)$, with $m_{L}+m_{H}=1$, as follows:

$$
\mathbb{E} D=\mu=S\left(m_{L}, m_{H}\right)
$$

where $0 \leq m_{L} \leq 1$ is the average fraction of total scale $S$ demanded by product $L$.

\subsection{Comparative Statics with respect to market scale and investment costs}

Given that the unit production and customization costs are non-negative, the expected ex-post costs scale up proportional with the market scale $S$. In contrast, the investment cost $I(Z)$ is independent of demand. Thus, as the market scale $S$ increases, the entire tradeoff curve scales up as in Figure 2. Given that its slope scales accordingly, the optimal platform strategy will "move down" the tradeoff curve as scale increases. Note that the sequence of optimal single-platform strategies will follow the investment cost ranking $0<I_{L}<I_{M}<I_{H}<I_{U}$ but it is possible that the two combined platform strategies $L H$ and $L M$ fall in-between $H$ and $U$, and $M$ and $U$, respectively. Given that platform strategy $L H$ incurs the lowest production customization costs, it becomes optimal as market scale $S$ is sufficiently large. The single flexible platform strategies $M$ and $U$ are optimal for intermediate scale. Exactly the same dynamics happen when all investment costs are scaled down by a common factor. 


\subsection{Comparative Statics with respect to market mix}

The expected production customization costs for a given strategy $Z$ are linear in demand mix while the investment cost are independent of the mix. At the boundary points when the market mix is concentrated on the lower $\left(m_{L}=1\right)$ or higher end product $\left(m_{H}=1\right)$, the respective optimal platform strategy always equals $L$ and $H$, independent of the scale. For interior mix $0<m_{L}<1$, the expected ex-post costs may increase or decrease with mix $m_{L}$, depending on the unit production customization costs for the low and high-end product. Figure 3 shows how the trade-off curve moves up and to the right as the mix changes from the low product $\left(m_{L}=1\right)$ to equal mix $\left(m_{L}=0.5\right)$ to the high product $\left(m_{L}=0\right)$. Typically, the customization cost for the single specialized platform strategies $L$ and $H$ are more sensitive to the scope $\left(m_{L}, m_{H}\right)$ then for the single flexible platform strategies $M$ and $U$ which gain in importance for intermediate mix values.

\section{Optimal platform strategy regions in partitioned demand space}

\subsection{Defining the optimal platform strategy based on expected demands}

Using (11), we can determine the lowest cost strategy $Z^{*}$ for a specific expected demand $\mathbb{E} D$. The set of expected demand vectors for which a given strategy $Z$ is optimal is a polyhedron (as it is the solution of a linear program). We can thus partition the expected demand space in different polyhedral or $\Omega_{Z}$-regions, where we define $\Omega_{Z}$ as the region where platform investment strategy $Z \in \mathcal{Z}=\{L, M, H, U, L M, L H\}$ (the set of 6 possible platform strategies) yields minimum costs. To define these $\Omega_{Z}$-regions, let us denote $\Delta_{z_{1}, z_{2}}=\mathcal{C}\left(z_{1}\right)-\mathcal{C}\left(z_{2}\right)$ as the difference in total cost $\mathcal{C}$ of the two platform strategies $z_{1}$ and $z_{2}$, with $z_{1}, z_{2} \in \mathcal{Z}$. Note that all $\Delta_{z_{1}, z_{2}}$ are linear equations in expected demand $\mu$. Where the total cost of two platform strategies is equal $\left(\Delta_{z_{1}, z_{2}}=0\right)$ a firm is indifferent between the two platform strategies $z_{1}$ and $z_{2}$, which we denote by the indifference line

$y_{z_{1}, z_{2}}$ (in Appendix all 15 possible indifference lines are expressed). Note that $y_{z_{1}, z_{2}}=y_{z_{2}, z_{1}}$. Given $\Delta_{z_{1}, z_{2}}=\mathcal{C}\left(z_{1}\right)-\mathcal{C}\left(z_{2}\right), \forall z_{1}, z_{2} \in \mathcal{Z}=\{L, M, H, U, L M, L H\}$, we propose that:

Proposition 2. Platform investment strategy $Z \in \mathcal{Z}$ is optimal for all mean demand vectors $\mu \in \Omega_{Z}$ where:

$$
\Omega_{Z}=\left\{\mu: \Delta_{Z, z_{2}} \leq 0 \quad \forall z_{2} \in \mathcal{Z}: z_{2} \neq Z\right\}
$$

By definition, the $\Omega_{Z}$-regions are convex polyhedra formed by the intersections of convex halfplanes. Of course, depending on the parameter values (i.e., different cost environments), some of the $\Omega_{Z}$-regions may change shape or be empty sets. The latter means platform strategy $Z$ is never optimal, as illustrated in section 5.2. Note that the size of the $\Omega_{Z}$-region is a metric for the robustness of the optimality for platform $Z$ : indeed $Z$ remains optimal for any forecast error $\varepsilon$ that keeps $\mu+\varepsilon \in \Omega_{Z}$.

Figure 4 visualizes a partitioning of the mean demand space when parameters are such that all $6 \Omega_{Z}$-regions are non-empty (Proposition 2 holds for all $Z \in \mathcal{Z}$ ). It also shows all 15 indifference 


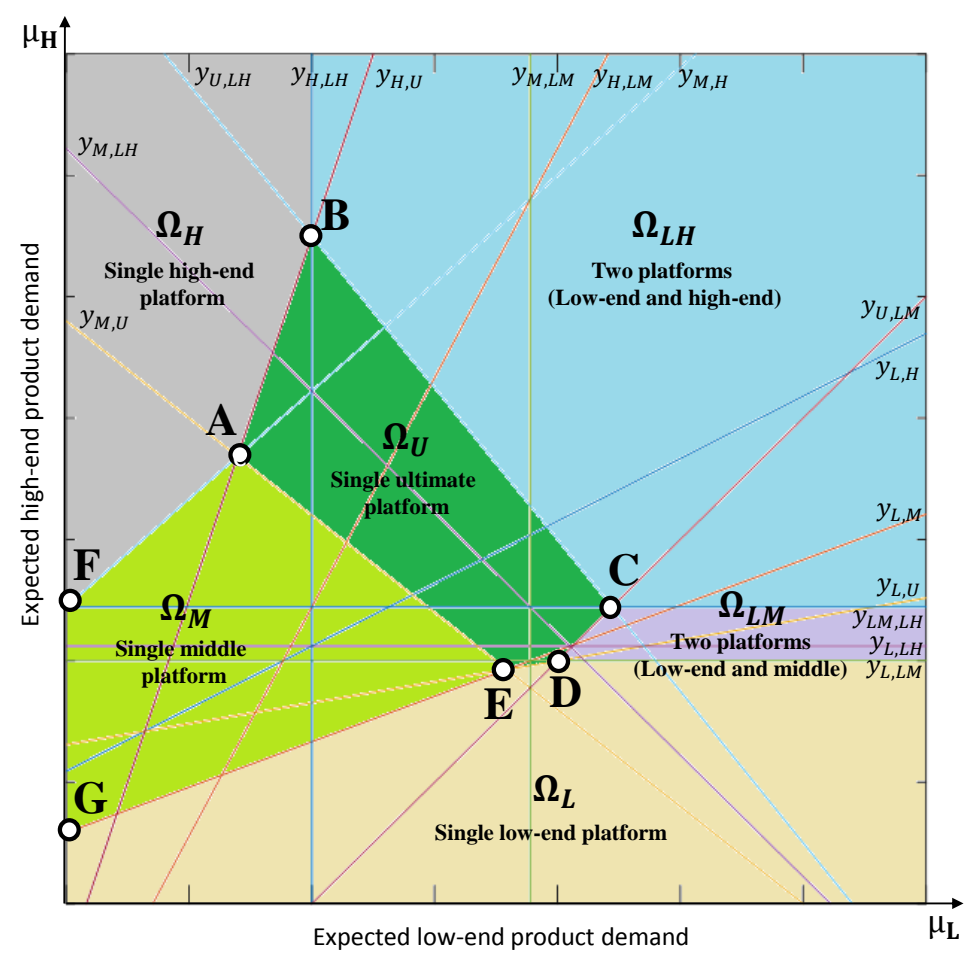

Figure 4: The space $\mathbb{R}_{+}^{2}$ of expected demands can be partitioned in polyhedral regions $\Omega_{Z}$ that uniquely define the optimal (lowest cost) platform strategy $Z$ if expected demand $\left(\mu_{L}, \mu_{H}\right) \in \Omega_{Z}$. Depending on the cost environment the size of the $\Omega_{Z}$-regions can change and/or an $\Omega_{Z}$-region can cease to exist.

lines $y_{z_{1}, z_{2}}=\left\{\mu: \Delta_{z_{1}, z_{2}}=0\right\}, \forall z_{1}, z_{2} \in \mathcal{Z}$. In that case, the entire mean demand space partitioning can be described by the 7 points $A$ to $G$ whose expressions are given in closed form in Appendix (e.g., point $A$ is defined by $\mathcal{C}(M)=\mathcal{C}(H)=\mathcal{C}(U)$ and its corresponding indifference lines $y_{M, U}=$ $\left.y_{H, U}=y_{M, H}\right)$. Notice that these seven points, forming the vertices of the $\Omega_{Z}$-regions, solely depend on investment and customization costs but are independent of the demand scale or mix.

From Figure 4 we find that, when demand for both products is sufficiently high so that $\mu \in \Omega_{L H}$, it is optimal to simultaneously develop platform $L$ and $H$. The higher the product demand, the more opportunity to leverage the high platform investment costs and the more important it is to have a lower unit production customization cost. This means that, when demand for both products is high, there is less benefit of platform commonality. Specialized platform strategies are optimal when the demand for either the low- or high-end product is sufficiently high (regions $\Omega_{L}$ and $\Omega_{H}$ ). Single flexible platform strategies are optimal when the demand for either or both the low- and high-end product is medium high (regions $\Omega_{M}$ and $\Omega_{U}$ ). Developing multiple platforms can be optimal with high demand for the low-end product, combined with medium to high demand for the high-end product (i.e., in the demand space regions $\Omega_{L H}$ and $\Omega_{L M}$ ).

These results shows that: 
Table 1: The data we use to illustrate different cost environments

\begin{tabular}{lccccc} 
Environment & Platform $i=$ & $L$ & $M$ & $H$ & $U$ \\
\hline (a) Low investment cost & $I_{i}$ & 0.255 & 0.36 & 0.525 & 0.54 \\
(b) Medium investment cost & $I_{i}$ & 0.51 & 0.72 & 1.05 & 1.08 \\
(c) High investment cost & $I_{i}$ & 0.85 & 1.20 & 1.75 & 1.80 \\
\hline (d) High flexibility cost of platform $M$ & $I_{i}$ & 0.51 & 0.935 & 1.05 & 1.08 \\
(e) High flexibility cost of platform $U$ & $I_{i}$ & 0.51 & 0.72 & 1.05 & 1.264 \\
\hline Unit production cost & $c_{i}$ & 0.10 & 0.15 & 0.20 & 0.25 \\
\hline Unit customization cost & $c_{i L}$ & 0 & 0.40 & 0.75 & 0.10 \\
& $c_{i H}$ & 1.75 & 0.50 & 0 & 0.15 \\
\hline
\end{tabular}

Corollary 1. Investment in single flexible platform strategies $M$ and $U$ can be optimal, even without taking uncertainty into consideration.

In the next section, we show that a change of the cost environment can lead to a change in shape of an $\Omega_{Z}$-region; when an $\Omega_{Z}$-region becomes an empty set we will informally say that 'the $\Omega_{Z}$-region ceases to exist'.

\subsection{Impact of the costs on optimal platform decisions}

Table 1 provides numerical data of different cost environments. We will illustrate how the cost environment impacts the $\Omega_{Z}$-regions. We first model the three environments (a), (b) and (c) of Table 1 to show how the size of the $\Omega_{Z}$-regions can change. This leads to a different optimal investment strategy for a given demand forecast. This is based on the development intensity of the platforms, which we quantify by the ratio of the investment in development versus the production customization costs:

$$
\text { development-intensity of platforms }=\frac{\sum_{Z \in \mathcal{Z}} I(Z)}{\sum_{Z \in \mathcal{Z}}\left(c_{L}(Z)+c_{H}(Z)\right)} .
$$

Figure 5 shows the corresponding $\Omega_{Z}$-regions and the expected demand vector, which is normalized to $(1,1)$ and represented by the black dot. As the development intensity increases, the regions $\Omega_{U}$ and $\Omega_{M}$ shift upward and thus become optimal. Indeed, given that forecasted demand $\left(\mu_{L}, \mu_{H}\right)$ remains the same, as the development intensity increases, the region where $L H$ is optimal shrinks to when demand $\mu_{L}$ and $\mu_{H}$ are both high, and the single platform strategies $M$ and $U$ have significant gain when both demand $\mu_{L}$ and $\mu_{H}$ are medium to low. In this situation, single platform strategy $H(L)$ also gains its optimal region when $\mu_{H}$ is much higher than $\mu_{L}$ ( $\mu_{L}$ is much higher than $\left.\mu_{H}\right)$.

When we consider environments (d) and (e) in Table 1 which are adjustments to environment (b) where the investment in the flexible platforms $M$ and $U$ increases while the investment cost of the other platforms remains unchanged, respectively $\Omega_{M}$ and $\Omega_{U}$ become empty sets (illustrated in the left and middle panel of Figure 6). Consequently, these platform strategies are no longer part of the trade-off curves (right panel of Figure 6). 

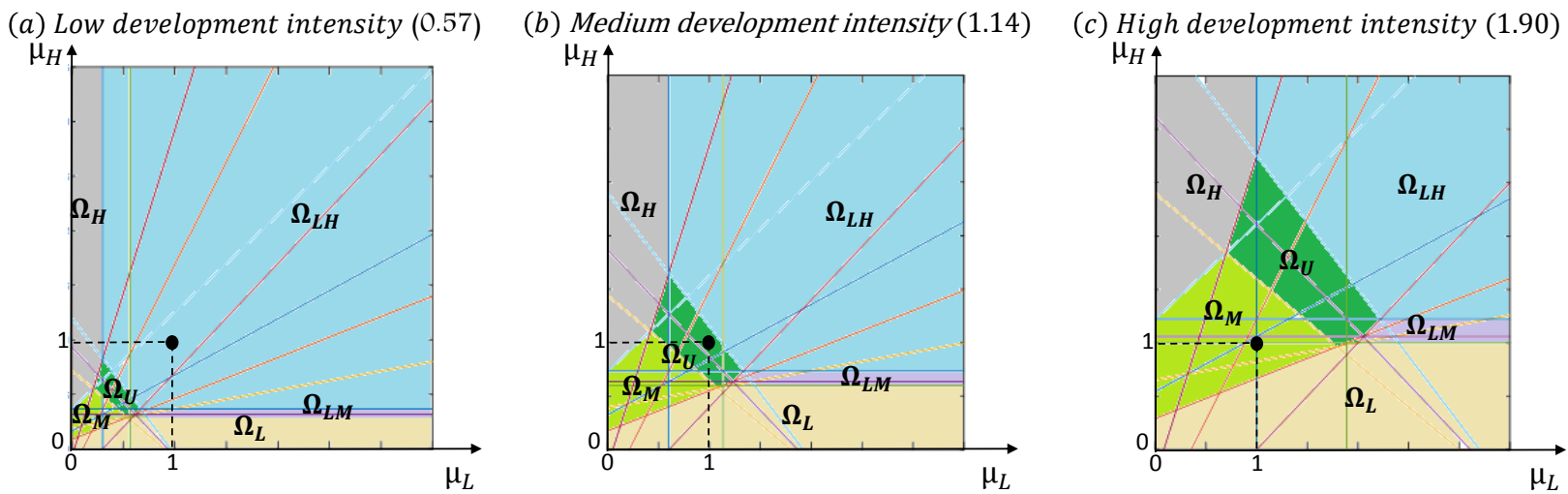

Figure 5: Given that forecasted demand $\left(\mu_{L}, \mu_{H}\right)$ remains the same, as the development intensity increases (from left to right panel, corresponding to environment (a) to (c) in Table 1), the region where $L H$ is optimal shrinks to when demand $\mu_{L}$ and $\mu_{H}$ are both high, and the single platform strategies $M$ and $U$ have significant gain when both demand $\mu_{L}$ and $\mu_{H}$ are medium to low. In this situation, single platform strategy $H(L)$ also gains its optimal region when $\mu_{H}$ is much higher than $\mu_{L}\left(\mu_{L}\right.$ is much higher than $\left.\mu_{H}\right)$.

We can find the maximal or threshold platform investment cost for which $\Omega_{M}$ and $\Omega_{U}$ become empty sets with the expressions given in Appendix. These calculations are based on the intersection points $A$ to $G$ in Figure 4. We find that $I_{M}=0.935=\bar{I}_{M}$ and $I_{U}=1.264=\bar{I}_{U}$ (which we used to define cost environment (d) and (e)). Notice that these threshold costs depend solely on investment and production customization costs but are independent of the demand scale or mix.

\section{Platform development under forecast uncertainty: Regret}

The analysis up until now prescribes the optimal platform portfolio based on the forecasted mean demand vector $\mu$. When forecasts are highly accurate, the actual demand $D$ observed after the platform development will be close to the forecasted mean demand $\mathbb{E} D=\mu$. In the limit of perfect forecast accuracy, both coincide so that the platform development decision is made knowing the actual demand $D$. Effectively, this simplifies the stochastic two-stage problem (9) to a single-stage deterministic optimization problem where the optimal strategy for a known demand $D$, denoted $Z_{D}^{*}$, solves

$$
Z_{D}^{*}=\arg \min _{Z \in \mathcal{Z}} \mathcal{C}_{D}(Z) \text { where } \mathcal{C}_{D}(Z)=I(Z)+C(D, Z)
$$

The associated optimal cost under perfect information about the demand $D$, denoted as $\mathcal{C}_{D}^{*}=$ $\mathcal{C}_{D}\left(Z_{D}^{*}\right)$, cannot be worse than the cost when using the platform $Z^{*}$ that is optimal in expectation:

$$
\mathcal{C}_{D}^{*}=\mathcal{C}_{D}\left(Z_{D}^{*}\right) \leq \mathcal{C}_{D}\left(Z^{*}\right)
$$



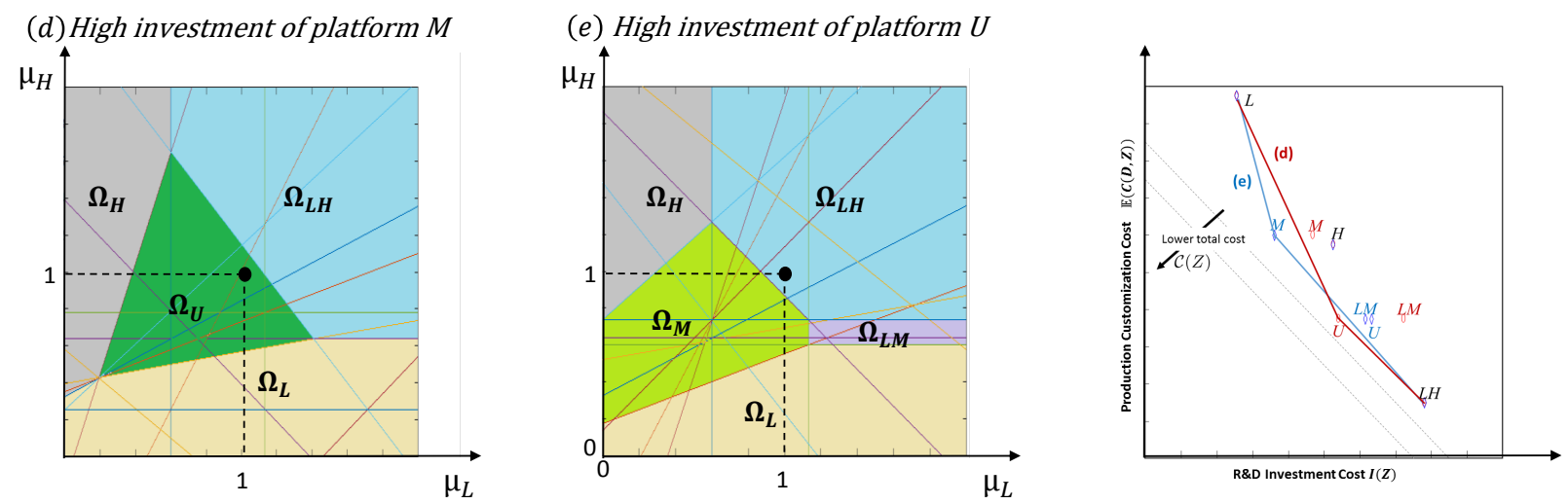

Figure 6: As the investment costs of the flexible platforms $M$ and $U$ increase beyond their thresholds (left and middle panel, respectively) while the other costs remain unchanged, their corresponding regions $\Omega_{M}$ and $\Omega_{U}$ cease to exist and the flexible platform strategies $M$ and $U$ cannot be optimal. Consequently, those strategies fall off their trade-off curves (right panel). The plots use costs (d) and (e) in Table 1 with normalized demand $\left(\mu_{L}, \mu_{H}\right)=(1,1)$ shown by a black dot.

Taking expectations directly yields a lower bound on the optimal cost under forecast uncertainty:

$$
\mathbb{E} \mathcal{C}_{D}^{*} \leq \mathcal{C}\left(Z^{*}\right)
$$

When demand forecasts are uncertain, the actual demand $D$ that is observed after platform investment can differ substantially from the forecasted mean demand $\mathbb{E} D=\mu$. In theory, when the decision maker is risk-neutral, such deviations are inconsequential in our model as the ex-ante objective (9) only depends on the forecasted mean demand, as shown in (11). In practice, however, managers may be concerned about how well their chosen platform performs when the actual demand deviates from the forecasted mean demand. In this paper, we analyze such concerns in two ways: In this section we investigate the "regret," defined as the "cost" of a wrong forecast:

$$
\text { Regret } R(D)=\mathcal{C}_{D}\left(Z^{*}\right)-\mathcal{C}_{D}\left(Z_{D}^{*}\right) \text { and } R=\mathbb{E} R(D)=\mathcal{C}\left(Z^{*}\right)-\mathbb{E C}_{D}^{*}
$$

Regret $R(D)$ is the cost that could have been avoided if we had known the actual demand $D$ instead of the demand forecast when making the platform decision. The expected regret equals the value of postponing the platform investment until forecasts become accurate (in the extreme case until demand is known) and is also referred to as the expected value of perfect information or the cost of uncertainty. Notice that, by definition, the optimal platform $Z^{*}$ is the platform that minimizes expected regret. In addition to a regret analysis, section 7 will analyze the concern of "making the wrong decision" by assuming that the decision maker is risk-averse using a mean-variance formulation.

Given that regret (i.e., the value of perfect information) depends on the cost parameters and on the entire shape of the demand distribution, analytical results for a general distribution are 
elusive. Therefore, we will focus on the celebrated normal distribution. Given that demands are non-negative, we truncate both bivariate distributions to the positive quadrant. To focus on the impact of uncertainty, we also will normalize the mean to $(1,1)$. Moreover, to analytically investigate the role of correlation for the truncated normal distributions, we restrict the domain of the distributions to the square $[0,2]^{2}$. Perfect correlation then implies a support on the lines $D_{H}=D_{L}$ and $D_{H}=2-D_{L}$ which is tractable. Specifically, the probability density of the normalized bivariate normal distribution is:

$$
h(D, \alpha, \rho)=\frac{g(D, \alpha, \rho)}{\int_{0}^{2} \int_{0}^{2} g(D, \alpha, \rho) \mathrm{d} D_{L} \mathrm{~d} D_{H}} \quad \forall D \in[0,2]^{2}
$$

where $g$ is the familiar normal probability density function with standard deviation $\sigma>0$ and correlation coefficient $\rho \in\{-1,1\}$ :

$$
g(D, \alpha, \rho)=\frac{1}{2 \pi \sigma^{2} \sqrt{1-\rho^{2}}} \exp \left(-\frac{\left(D_{L}-1\right)^{2}-2 \rho\left(D_{L}-1\right)\left(D_{H}-1\right)+\left(D_{H}-1\right)^{2}}{2 \sigma^{2}\left(1-\rho^{2}\right)}\right) .
$$

Next, we analytically investigate regret when demand is normally distributed over the square $[0,2]^{2}$ and perfectly correlated $\rho= \pm 1$ and conjecture that under high demand uncertainty, the expected regret is higher for negative than for positive demand correlation. Later, we numerically investigate regret for general cost and uncertainty parameters to verify under which conditions this conjecture is supported.

\subsection{Regret with perfectly correlated demand}

With perfect correlation, the support of our normalized demand distribution becomes one-dimensional. If $\rho=+1, D_{H}=D_{L}$ so that the product mix remains constant. If $\rho=-1, D_{L}+D_{H}=2$ and the scale remains constant. The actual cost for platform strategy $Z$ as specified by (8) reduces to:

$$
\mathcal{C}_{D}(Z)= \begin{cases}I(Z)+D_{L}\left(c_{L}(Z)-c_{H}(Z)\right)+2 c_{H}(Z) & \text { if } \rho=-1 \\ I(Z)+D_{L}\left(c_{L}(Z)+c_{H}(Z)\right) & \text { if } \rho=+1\end{cases}
$$

With perfect correlation, the actual cost $\mathcal{C}_{D}(Z)$ thus is a univariate function of $D_{L}$, as shown in Figure 7. For perfectly positive correlation (right panel), for any platform $Z$, the cost $\mathcal{C}_{D}(Z)$ is non-decreasing in $D_{L}$, which cannot be guaranteed for perfectly negative correlation (left panel). The regret $R\left(D_{L}\right)$ is the cost that could have been avoided by using platform $Z_{D_{L}}^{*}$ (traced in green) instead of $Z^{*}$ (in red). When the realized demand $D_{L}$ equals the forecasted mean $\mu_{L}=1$, the regret is zero because both the ex-ante platform decision $Z^{*}$, which minimizes $I(Z)+c_{L}(Z)+c_{H}(Z)$, coincides with the postponed platform $Z_{D_{L}=\mu_{L}}^{*}$. This holds for as long $D_{L} \in \Omega_{Z^{*}}$, where the $\Omega_{Z^{-}}$ regions simplify to intervals, as shown on the horizontal axes in Figure 7 . These intervals coincide with the vertical projections of the convex hull that traces the minimal cost curves $\mathcal{C}_{D}(Z)$, shown 

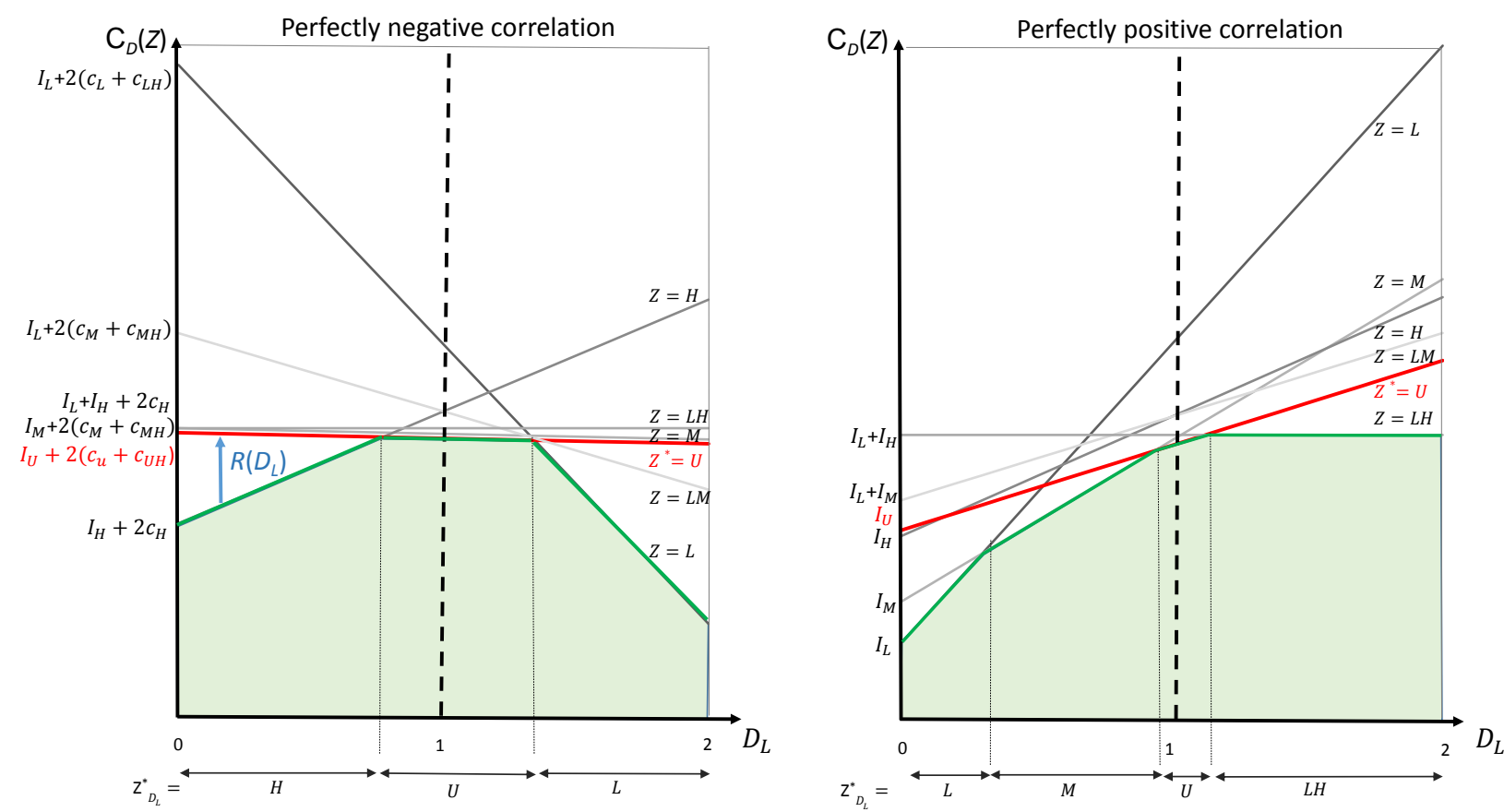

Figure 7: With perfect correlation (negative left panel; positive right panel), the actual cost $\mathcal{C}_{D}(Z)$ is a univariate function of $D_{L}$. The regret $R\left(D_{L}\right)$ is the cost that could have been avoided by using platform $Z_{D_{L}}^{*}$ (denoted on horizontal axis and its cost traced in green) instead of $Z^{*}$ (in red).

in bold green. We denote the demand intervals where platform strategy $Z$ is optimal as:

$$
\left[D_{L}^{\min }(Z), D_{L}^{\max }(Z)\right]=\left\{D_{L} \in[0,2]: Z_{D_{L}}^{*}=Z\right\}
$$

(If $D_{L}^{\min }(Z)>D_{L}^{\max }(Z)$, then platform strategy $Z$ is never optimal.)

With these intervals in hand, the average regret is obtained by integrating using the normalized probability density $p\left(D_{L}\right)$ of the single dimensional demand $D_{L}$ :

Corollary 2. With perfectly correlated demand, the expected regret $R$

$$
R=\min _{Z \in \mathcal{Z}}\left\{I(Z)+c_{L}(Z)+c_{H}(Z)\right\}-\sum_{Z \in \mathcal{Z}} \int_{D_{L}^{\min }(Z)}^{D_{L}^{\max }(Z)} p\left(D_{L}\right) \mathcal{C}\left(D_{L}, Z\right) d D_{L} .
$$

General closed form expressions for the expected regret are elusive as the intervals are dependent on the relative values of investment and customization costs. Only for $D_{L}=0$ is the optimal postponed strategy fixed: For perfectly positive correlation, $Z_{D_{L}=0}^{*}=L$ because when scale is zero the total cost reduces to $I(Z)$, which is minimized by platform $L$. (At the other end of the spectrum, we know that $\left.\mathcal{C}_{D_{L}=1}(Z) \leq I_{L}+I_{H}\right)$. Similarly, for perfectly negative correlation, $Z_{D_{L}=0}^{*}=H$ because when $D_{L}=0$, the optimal postponed platform strategy minimizes $I(Z)+2 c_{H}(Z)$, which is platform $H$. 
Corollary 2 does provide direct insight into the sensitivity of the expected regret to cost parameters and demand correlation and variability. For example, the regret is equally impacted by a local change in the cost parameters of the postponed-optimal platforms $\left(Z_{D_{L}}^{*}: D_{L} \in[0,2]\right)$ (as that moves the convex hull that traces the minimal cost curves $\mathcal{C}_{D}(Z)$ ), while it is locally independent of the other platforms. Also, the expected regret is (weakly) increasing in demand variance. Last, and most interesting, is the sensitivity to correlation: notice that all 6 platforms have (weakly) upward sloping cost lines for $\rho=+1$ while at least one platform $(L)$ has a downward sloping cost line for $\rho=-1$. Thus, the slopes of the convex hull that traces the minimal cost curves $\mathcal{C}_{D}(Z)$ typically vary more for negative than positive correlation. Given that the slope of the optimal ex-ante platform's cost $\mathcal{C}_{D}\left(Z^{*}\right)$ remains constant, it means that as $D_{L}$ goes from 0 to 2 the regret $R\left(D_{L}\right)$ varies more for negative than positive correlation. This suggest that, if the demand distribution of $D_{L}$ has sufficient variability so that the intervals beyond $\left[D_{L}^{\min }\left(Z^{*}\right), D_{L}^{\max }\left(Z^{*}\right)\right]$ have positive probability, then:

Conjecture 1. For high uncertainty, the expected regret is higher for negative than for positive correlation.

In the next subsection, we shall confirm Conjecture 1 numerically for a test bed of parameters that covers most plausible values under the normalized bivariate normal distribution.

Conjecture 1 is striking because it means that the expected regret or value of perfect information when investing in platform flexibility is higher under negative correlation. This emphasizes that forecast accuracy for platform development is more important for negatively correlated demands. In manufacturing flexibility studies the opposite result is often seen: thanks to risk pooling under negative correlation the expected regret is lower under negative correlation.

\subsection{Regret with bivariate normally distributed demand}

In this section we numerically analyze the expected regret $R$ assuming that demand is normally distributed over the square $[0,2]^{2}$. To calculate the expected regret, the two-dimensional integration was approximated by a summation over 500,000 sample demand points drawn from the distribution. This allows us to investigate how $R$ depends on correlation, variance, and cost parameters.

To investigate how Conjecture 1 depends on the cost parameters, we performed a comprehensive numerical analysis over the following test bed of parameters that covers most plausible values. The four investment cost parameters $\left(I_{L}, I_{M}, I_{H}, I_{U}\right)$ range from 0 to 10 using a step size 1 , the four production cost parameters $\left(c_{L}, c_{M}, c_{H}, c_{U}\right)$ range from 0 to 6 using a step size 1 , and the 6 customization cost parameters $\left(c_{L H}, c_{H L}, c_{M L}, c_{M H}, c_{U L}, c_{U H}\right)$ range from 0 to 8 using a step size 1. Given our model assumptions (e.g., $\left.I_{L}<I_{M}<I_{H}<I_{U}\right)$ and changing $\rho$ from -1 to 1 with step size 1 and $\sigma$ from 0.1 to 1 with step size 0.1 , our test bed contains 462,600 parameter scenarios. Given that there are 30 combinations of $\rho$ and $\sigma$ that are distribution driven, there are $462,600 / 30=15,420$ different cost parameter scenarios.

Table 2 shows that Conjecture 1 holds more often as $\sigma$ increases. It confirms that Conjecture 1 
Table 2: Investigation of Conjecture 1 and summary results of the expected regret over a testbed of 15,420 scenarios of the cost parameters.

\begin{tabular}{c|c|ccc}
\hline \multirow{2}{*}{$\sigma$} & Number of scenarios & \multicolumn{3}{|c}{ Average expected regret $R$} \\
\cline { 3 - 5 } & confirming Conjecture 1 & $\rho=-1$ & $\rho=0$ & $\rho=+1$ \\
\hline 0.1 & $94.37 \%$ & 0.025 & 0.019 & 0.018 \\
0.2 & $100.00 \%$ & 0.213 & 0.101 & 0.061 \\
0.3 & $100.00 \%$ & 0.641 & 0.323 & 0.139 \\
0.4 & $100.00 \%$ & 1.141 & 0.618 & 0.233 \\
0.5 & $100.00 \%$ & 1.546 & 0.871 & 0.312 \\
0.6 & $100.00 \%$ & 1.839 & 1.054 & 0.369 \\
0.7 & $100.00 \%$ & 2.042 & 1.185 & 0.410 \\
0.8 & $100.00 \%$ & 2.177 & 1.273 & 0.439 \\
0.9 & $100.00 \%$ & 2.286 & 1.343 & 0.458 \\
1 & $100.00 \%$ & 2.360 & 1.389 & 0.474 \\
\hline
\end{tabular}

requires the qualifier "under high uncertainty" where the exact quantification of that uncertainty threshold will be distribution dependent. For a coefficient of variation of 0.2 and higher, Conjecture 1 is supported in all of the instances tested. The three columns on the right in Table 2 also confirm that average expected regret over the testbed scenarios is higher as correlation $\rho$ is more negative and demand variance $\sigma$ increases.

\section{Platform development under forecast uncertainty: Risk-Aversion}

Instead of expected regret, an alternative approach to model managers' concerns about how well their ex-ante chosen platform performs when the actual demand deviates from the forecasted mean demand is to assume that the decision maker is risk-averse. The optimal platform $Z^{*}$ is our prescription for a risk-neutral decision maker. In this section we investigate the best platform prescription $Z^{M V}$ for a risk-averse decision maker who maximizes the celebrated mean-variance utility. (Mean-variance utility formations are used in financial-portfolio theory, as reviewed by pioneer Markowitz (1991), and have been adopted in addition to other concave utility functions in newsvendor networks; see Van Mieghem (2003) and references therein.)

Using a mean-variance formulation, the risk-averse decision maker maximizes

$$
U^{M V}(Z)=\pi(Z)-\frac{\gamma}{2} \sigma^{2}(Z)
$$

over all platform strategies $Z \in \mathcal{Z}$ where $\gamma \geq 0$ is the decision-maker's coefficient of risk-aversion (the larger $\gamma$ the more risk averse), $\pi(Z)$ is the expected profit and $\sigma^{2}(Z)$ the variance of the profit. In essence, risk-averse decision makers dislike profit variability, which is modeled with a penalty for profit variance. With exogenous demand, revenues are independent of the platform and customization decisions. Given that utilities are invariant to translations, we can subtract the constant revenues and convert the maximization problem into the following minimization problem. 


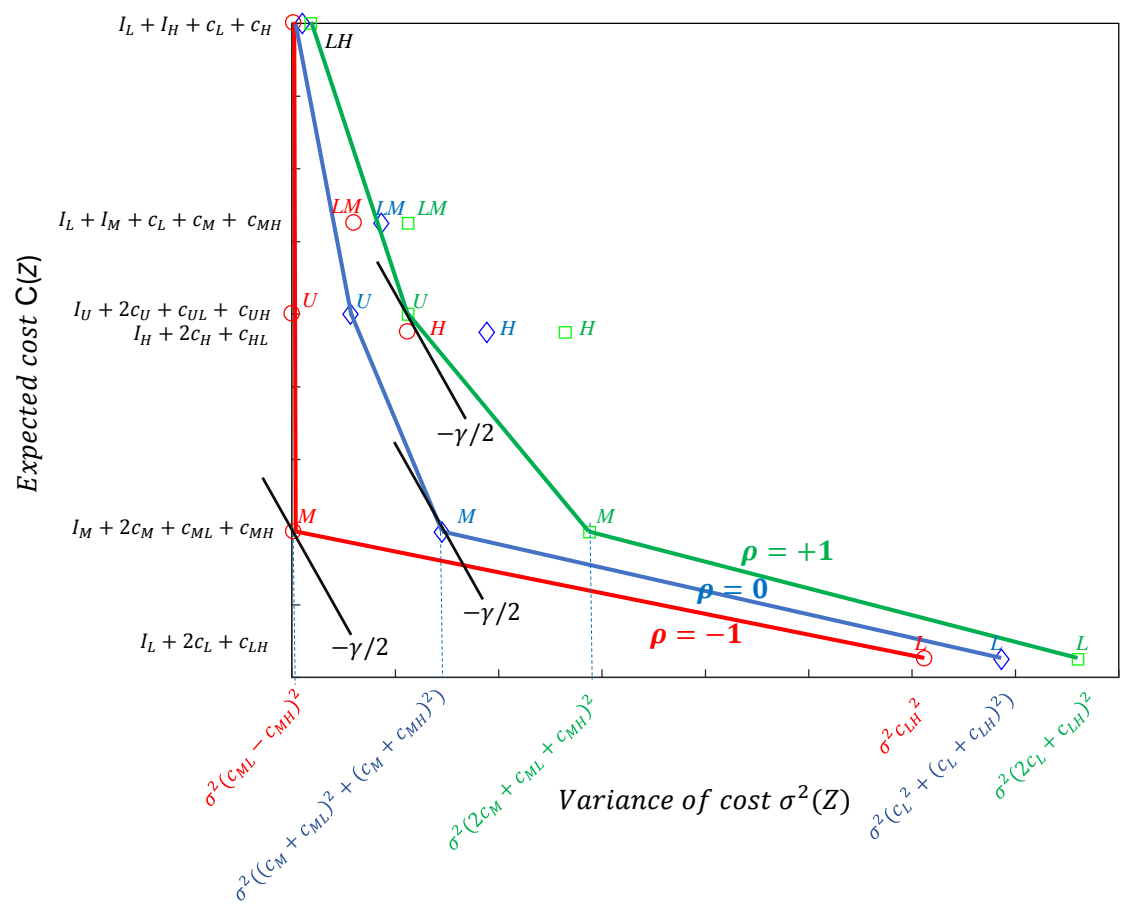

Figure 8: The mean-variance frontier for $\mu \in[1,1]$ and $\rho \in\{-1,0,+1\}$ depends on the investment and production customization cost, the demand variance $\sigma^{2}$ and the correlation $\rho$. With coefficient of risk-aversion $\gamma$ the operational hedging platform strategy is $U$ for $\rho=+1$ and $M$ for $\rho=0$ or -1 ; note that platform $M$ is a perfect hedge for $\rho=-1$.

Find the platform $Z^{M V}$ for the risk mean-variance platform decision maker that minimizes

$$
Z^{M V}=\arg \min _{Z \in \mathcal{Z}} \mathcal{C}^{M V}(Z) \text { where } \mathcal{C}^{M V}(Z)=\mathcal{C}(Z)+\frac{\gamma}{2} \mathbb{E}\left[C_{D}(Z)-\mathcal{C}(Z)\right]^{2}
$$

where the expected $\operatorname{cost} \mathcal{C}(Z)=\mathbb{E} C_{D}(Z)$ and $\mathcal{C}_{D}(Z)=I(Z)+C(D, Z)$, as defined by (14). The profit variance equals the cost variance which simplifies to:

$$
\begin{aligned}
\sigma^{2}(Z) & =\mathbb{E}\left[\left(D_{L}-\mu_{L}\right) c_{L}(Z)+\left(D_{H}-\mu_{H}\right) c_{H}(Z)\right]^{2} \\
& =\sigma_{L}^{2} c_{L}^{2}(Z)+2 \rho \sigma_{L} \sigma_{H} c_{L}(Z) c_{H}(Z)+\sigma_{H}^{2} c_{H}^{2}(Z) \\
& =\sigma^{2}\left(c_{L}^{2}(Z)+2 \rho c_{L}(Z) c_{H}(Z)+c_{H}^{2}(Z)\right) \quad \text { if } \sigma_{H}=\sigma_{L}=\sigma,
\end{aligned}
$$

where $\sigma_{i}$ is the standard deviation of $D_{i}$.

The solution to (24) is easily found in two steps: First, construct the mean-variance frontier, which is the "south-western part" of the convex hull of the set $\left\{\left(\mathcal{C}(Z), \sigma^{2}(Z)\right): Z \in \mathcal{Z}\right\}$. Second, find the platform $Z^{M V}$ as the platform on the frontier where the frontier's (sub)gradient equals $-\gamma / 2$. In other words, as risk aversion increases, more expensive platforms higher on the frontier will be preferred. This process is illustrated for symmetric normalized demand $(\mu=[1,1]$ and 
$\left.\sigma_{L}=\sigma_{H}=\sigma\right)$ in Figure 8 for investment intensive platforms (i.e., $I(Z)$ is the main driver for the expected cost rather than the customization cost). According to the parameters of that figure, a risk-neutral $(\gamma=0)$ investor should invest in platform $L$. As $\gamma$ increases beyond the slope (leftderivative) of the frontier at $L$, platform $M$ becomes optimal and becomes the "first hedge." As $\gamma$ increases beyond the slope of the frontier at $M$, platform $U$ becomes optimal (and is the "second hedge"). Finally, as $\gamma$ increases beyond the slope of the frontier at $U$, the combined platform strategy $L H$ becomes optimal and remains optimal for higher levels of risk aversion (because it has the lowest cost variance).

Two observations follow from Figure 8. First, as correlation decreases, the efficient frontier moves to the left, in accordance with (27). Thus, the slope of the efficient frontier at the risk-neutral platform $Z^{*}$ decreases as correlation decreases. Therefore, a risk-averse decision maker can reduce risk more by increasing investment to the second platform on the frontier ( $M$ in Figure 8 ) under negative correlation than positive correlation. In other words, $M$ is a more effective operational hedge under negative correlation. This is good news as we established that the average regret is higher under negative correlation so that the "bang for the buck" is higher under negative correlation. Also, it takes a higher level of risk aversion (higher $\gamma$ ) under positive correlation for the second platform $M$ to be optimal. Note, however, that the efficient platforms (those on the frontier) for $\rho=-1$ are not necessarily the same as for $\rho=+1$ (that depends on the relative magnitudes of the ex-post production customization costs).

Second, as the demand becomes more variable ( $\sigma$ increases), the entire frontier is scaled to the right so that the frontier becomes less steep (while the order of efficient platform decisions remains unchanged). For a constant level of risk-aversion $\gamma$, efficient but higher investment cost platforms (flexible platforms $M$ and $U$ ) thus become more attractive and effective as operational hedges.

Figure 9 shows the mean variance frontier as the development intensity increases. As investment costs increase compared to production customization cost, the optimal platform for a risk aversion level $\gamma$, changes from $L H$ to $U$ to $M$, as we saw earlier in Figure 5, and eventually to the low-end platform $L$. As investment costs increase, the frontiers of-course move up but they also become steeper around the risk-neutral platform. Furthermore, while the single flexible platform strategies $M$ and $U$ do not have the lowest expected cost, they become attractive for a slightly risk-averse decision maker as good operational hedges: a small increase in expected cost greatly reduces cost variance. These findings suggest:

Conjecture 2. A flexible platform ( $M$ or $U$ ) is an optimal hedge for risk-averse decision makers in highly development-intensive environments with highly uncertain and more negatively-correlated demand.

This means that risk-averse decision makers should invest more than risk-neutral counterparts (in contrast to typical risk-averse newsvendors of a single product who invest less than the riskneutral newsvendor). 


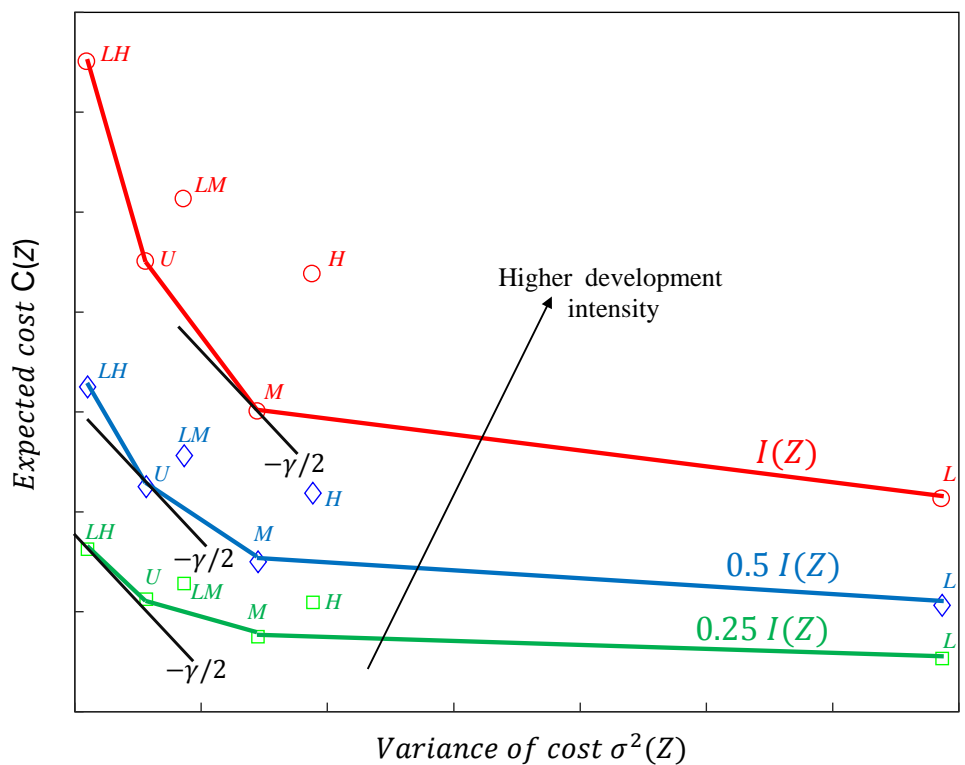

Figure 9: The mean-variance frontier moves up as the investment cost increases. (The figure assumes $\rho=0$ and $\sigma=1$ ).

\section{Conclusions}

In this paper we analyze which platforms a firm should develop to produce multiple products. As platform development often requires substantial $R \& D$ investments and future demand may vary both in scale (number of units sold) and scope (product mix), this decision may have large cost implications. The platform portfolio may consist of either specialized platforms, aligned with the functionalities of the products, or may retain some flexibility to cost-efficiently customize to either product variant. We characterize the optimal platform portfolio based on the investment versus production customization tradeoff and the estimated future product demand. We also incorporate risk evaluations by evaluating the regret of developing a sub-optimal platform portfolio and the variance of the future production customization costs. Our analysis may support companies in the development of their platform portfolio. Future research could be devoted to a dynamic analysis of flexible platform developments over time.

\section{References}

Alptekinoglu, Aydin, Karthik Ramachandran. 2015. Flexible products for dynamic preferences. Working paper, available at SSRN 2037631.

Bassamboo, Achal, Ramandeep S Randhawa, Jan A Van Mieghem. 2010. Optimal flexibility configurations in newsvendor networks: Going beyond chaining and pairing. Management Science 56(8) 1285-1303. 
Bhaskaran, Sreekumar R, Karthik Ramachandran. 2011. Managing technology selection and development risk in competitive environments. Production and Operations Management 20(4) $541-555$.

Boonman, Hendrika Jobina, Verena Hagspiel, Peter M Kort. 2015. Dedicated vs product flexible production technology: Strategic capacity investment choice. European Journal of Operational Research 244(1) 141-152.

Boute, Robert, Maud Van den Broeke, Kristof Deneire. 2018. Barco implements platform-based product development in its healthcare division. Interfaces 48(1) 35-44.

Chandler, Alfred Dupont, Takashi Hikino, Alfred D Chandler. 2009. Scale and scope: The dynamics of industrial capitalism. Harvard University Press.

Chen, Yuwen, Asoo J Vakharia, Aydin Alptekinolu. 2008. Product portfolio strategies: The case of multifunction products. Production and Operations Management 17(6) 587-598.

Childs, Paul D, Alexander J Triantis. 1999. Dynamic r\&d investment policies. Management Science 45(10) 1359-1377.

Chod, Jiri, Nils Rudi, Jan A Van Mieghem. 2010. Operational flexibility and financial hedging: Complements or substitutes? Management Science 56(6) 1030-1045.

Chronopoulos, Michail, Bert De Reyck, Afzal Siddiqui. 2011. Optimal investment under operational flexibility, risk aversion, and uncertainty. European Journal of Operational Research 213(1) 221237.

Desai, Preyas, Sunder Kekre, Suresh Radhakrishnan, Kannan Srinivasan. 2001. Product differentiation and commonality in design: Balancing revenue and cost drivers. Management Science 47(1) $37-51$.

Financial Times. 2012. Manufacturing: Hopes are pinned on flexbile modularity. March 62017 .

Financial Times. 2013. Building blocks to cut output costs. March 62017 .

Financial Times. 2015. Gm to put 5bn usd into emerging market car. July 282017.

Fine, Charles H, Robert M Freund. 1990. Optimal investment in product-flexible manufacturing capacity. Management Science 36(4) 449-466.

Goyal, Manu, Serguei Netessine. 2011. Volume flexibility, product flexibility, or both: The role of demand correlation and product substitution. Manufacturing \& Service Operations Management 13(2) 180-193.

Huchzermeier, Arnd, Christoph H Loch. 2001. Project management under risk: Using the real options approach to evaluate flexibility in r\&d. Management Science 47(1) 85-101. 
Jiao, Jianxin Roger, Timothy W Simpson, Zahed Siddique. 2007. Product family design and platform-based product development: a state-of-the-art review. Journal of intelligent Manufacturing 18(1) 5-29.

Kettunen, Janne, Yael Grushka-Cockayne, Zeger Degraeve, Bert De Reyck. 2015. New product development flexibility in a competitive environment. European Journal of Operational Research 244(3) 892-904.

Krishnan, Vish, Shantanu Bhattacharya. 2002. Technology selection and commitment in new product development: The role of uncertainty and design flexibility. Management Science 48(3) $313-327$.

Krishnan, Vish, Saurabh Gupta. 2001. Appropriateness and impact of platform-based product development. Management Science 47(1) 52-68.

Krishnan, Viswanathan, Karl T Ulrich. 2001. Product development decisions: A review of the literature. Management science 47(1) 1-21.

Lambert, Fred. 2017. Volvo credits tesla for creating ev demand, says they will stop developing diesel engines to focus on evs. https://electrek.co/2017/05/17/ volvo-tesla-says-stop-diesel-electric-vehicle/. [Online; accessed 17-05-2017].

Li, Zhongkai, Zhihong Cheng, Yixiong Feng, Jinyong Yang. 2013. An integrated method for flexible platform modular architecture design. Journal of Engineering Design 24(1) 25-44.

Madni, Azad M. 2012. Adaptable platform-based engineering: Key enablers and outlook for the future. Systems Engineering 15(1) 95-107.

Markowitz, Harry M. 1991. Foundations of portfolio theory. The journal of finance 46(2) 469-477.

Netessine, Serguei, Gregory Dobson, Robert A Shumsky. 2002. Flexible service capacity: Optimal investment and the impact of demand correlation. Operations Research 50(2) 375-388.

Robertson, David, Karl Ulrich. 1998. Planning for product platforms. MIT Sloan Management Review 39(4) 20.

Shumsky, Robert A, Fuqiang Zhang. 2009. Dynamic capacity management with substitution. Operations research $\mathbf{5 7 ( 3 )} 671-684$.

Simpson, Timothy W, J Jiao, Zahed Siddique, Katja Hölttä-Otto. 2014. Advances in product family and product platform design. New York: Springer .

Simpson, Timothy W, Jonathan R Maier, Farrokh Mistree. 2001. Product platform design: method and application. Research in engineering Design 13(1) 2-22.

Suh, Eun Suk, Olivier L De Weck, David Chang. 2007. Flexible product platforms: framework and case study. Research in Engineering Design 18(2) 67-89. 
Van den Broeke, Maud, Robert Boute, Brecht Cardoen, Behzad Samii. 2017. An efficient solution method to design the cost-minimizing platform portfolio. European Journal of Operational Research 259(1) 236-250.

Van den Broeke, Maud, Robert Boute, Behzad Samii. 2015. Evaluation of product-platform decisions based on total supply chain costs. International Journal of Production Research 53(18) $5545-5563$.

Van Mieghem, Jan A. 1998. Investment strategies for flexible resources. Management Science 44(8) 1071-1078.

Van Mieghem, Jan A. 2003. Capacity management, investment, and hedging: Review and recent developments. Manufacturing \& Service Operations Management 5(4) 269-302.

Van Mieghem, Jan A. 2007. Risk mitigation in newsvendor networks: Resource diversification, flexibility, sharing, and hedging. Management Science 53(8) 1269-1288.

Volvo. 2014. All-new xc90 the first volvo built on the company's new scalable product architecture. https://www.media.volvocars.com/global/en-gb/media/pressreleases/148966. [Online; accessed 02-04-2016].

Yang, Liu, Chi To Ng. 2014. Flexible capacity strategy with multiple market periods under demand uncertainty and investment constraint. European Journal of Operational Research 236(2) 511521.

Zhang, Linda L. 2015. A literature review on multitype platforming and framework for future research. International Journal of Production Economics 168 1-12. 


\section{Appendix}

\section{A Proof of Proposition 1 .}

We can distinguish between 15 possible platform investment strategies:

1. Platform strategy $\mathbf{L}\left(x_{L}=1\right)$ : A firm only develops the low-end platform at investment $\operatorname{cost} I_{L}$. In that case, both the low-end and high-end product are derived from this low-end platform. Hence, the associated total cost for this platform investment strategy is defined by: $\mathcal{C}(L)=c_{L H} \mu_{H}+c_{L}\left(\mu_{L}+\mu_{H}\right)+I_{L}$.

2. Platform strategy $\mathbf{H}\left(x_{H}=1\right)$ : only develop the high-end platform so that the high-end product is derived from its specialized platform and the low-end platform is derived from an over-designed platform. The associated total cost then equals: $\mathcal{C}(H)=c_{H L} \mu_{L}+c_{H}\left(\mu_{L}+\right.$ $\left.\mu_{H}\right)+I_{H}$.

3. Platform strategy $\mathbf{M}\left(x_{M}=1\right)$ : only develop the middle platform so that both the highend and low-end product are derive from this middle platform. The associated total cost then equals: $\mathcal{C}(M)=c_{M L} \mu_{L}+c_{M H} \mu_{H}+c_{M}\left(\mu_{L}+\mu_{H}\right)+I_{M}$.

4. Platform strategy $\mathbf{U}\left(x_{U}=1\right)$ : only develop the ultimate platform so that both the highend and low-end product are derive from this ultimate platform. The associated total cost then equals: $\mathcal{C}(U)=c_{U L} \mu_{L}+c_{U H} \mu_{H}+c_{U}\left(\mu_{L}+\mu_{H}\right)+I_{U}$.

5. Platform strategy LH $\left(x_{L}=1\right.$ and $\left.x_{H}=1\right)$ : invest in the development of a low-end and high-end platform and derive each product from its specialized platform. As $c_{L}+c_{L L}<$ $c_{H}+c_{H L}$ and $c_{H}+c_{H H}<c_{L}+c_{L H}$, it is optimal to derive the low-end product from the lowend platform and the high-end product from the high-end platform, so that $x_{L L}=x_{H H}=1$ and $x_{i j}=0$ otherwise. Hence $\mathcal{C}(L H)=c_{L} \mu_{L}+c_{H} \mu_{H}+I_{L}+I_{H}$.

6. Platform strategy LM $\left(x_{L}=1\right.$ and $\left.x_{M}=1\right)$ : invest in the development of a low-end and middle platform and derive the low-end product from its specialized platform and the highend product from the middle platform. As $c_{L}+c_{L L}<c_{M}+c_{M L}$ and $c_{M}+c_{M H}<c_{L}+c_{L H}$, it is optimal to derive the low-end product from its specialized platform and the high-end product from the middle platform, so that $x_{L L}=x_{M H}=1$ and $x_{M L}=x_{L H}=0$. Hence $\mathcal{C}(L M)=c_{M H} \mu_{H}+c_{L} \mu_{L}+c_{M} \mu_{H}+I_{L}+I_{M}$.

7. Platform strategy $\mathbf{M H}\left(x_{M}=1\right.$ and $\left.x_{H}=1\right)$ : invest in the development of a high-end and middle platform. As $c_{M}+c_{M L}<c_{H}+c_{H L}$, the low-end product is derived from the middle platform $\left(x_{M L}=1\right)$ and as $c_{H}+c_{H H}<c_{M}+c_{M H}$, the high-end product from the high-end platform $\left(x_{H H}=1\right)$. Hence $\mathcal{C}(M H)=c_{M L} \mu_{L}+c_{M} \mu_{L}+c_{H} \mu_{H}+I_{M}+I_{H}$. 
8. Platform strategy LU $\left(x_{L}=1\right.$ and $\left.x_{U}=1\right)$ : invest in the development of a low-end and ultimate platform. As $c_{L}+c_{L L}<c_{U}+c_{U L}$, the low-end product is derived from the low-end platform $\left(x_{L L}=1\right)$ and as $c_{U}+c_{U H}<c_{L}+c_{L H}$, the high-end product from the ultimate platform $\left(x_{U H}=1\right)$. Hence $\mathcal{C}(L U)=c_{U H} \mu_{H}+c_{L} \mu_{L}+c_{U} \mu_{H}+I_{L}+I_{U}$.

9. Platform strategy MU $\left(x_{M}=1\right.$ and $\left.x_{U}=1\right)$ : invest in the development of a middle and ultimate platform. As $c_{U}+c_{U L}<c_{M}+c_{M L}$ and $c_{U}+c_{U H}<c_{M}+c_{M H}$, both products are derived from the ultimate platform $\left(x_{U L}=1\right.$ and $\left.x_{U H}=1\right)$. Hence $\mathcal{C}(M U)=c_{U L} \mu_{L}+$ $c_{U H} \mu_{H}+c_{U}\left(\mu_{L}+\mu_{H}\right)+I_{M}+I_{U}$.

10. Platform strategy HU $\left(x_{H}=1\right.$ and $\left.x_{U}=1\right)$ : invest in the development of a high-end and ultimate platform. As $c_{U}+c_{U L}<c_{H}+c_{H L}$, the low-end product is derived from the ultimate platform $\left(x_{U L}=1\right)$ and as $c_{H}+c_{H H}<c_{U}+c_{U H}$, the high-end product from the high-end platform $\left(x_{H H}=1\right)$. Hence $\mathcal{C}(H U)=c_{U L} \mu_{L}+c_{U} \mu_{L}+c_{H} \mu_{H}+I_{H}+I_{U}$.

11. Platform strategy LMH $\left(x_{L}=1, x_{M}=1\right.$ and $\left.x_{H}=1\right)$ : invest in the development of a low-end, middle and high-end platform. As $c_{L}+c_{L L}<c_{M}+c_{M L}<c_{H}+c_{H L}$ and $c_{H}+c_{H H}<c_{M}+c_{M H}<c_{L}+c_{L H}$, both products are derived from their specialized platform $\left(x_{L L}=1\right.$ and $\left.x_{H H}=1\right)$. Hence $\mathcal{C}(L M H)=c_{L} \mu_{L}+c_{H} \mu_{H}+I_{L}+I_{M}+I_{H}$.

12. Platform strategy LHU $\left(x_{L}=1, x_{H}=1\right.$ and $\left.x_{U}=1\right)$ : invest in the development of a low-end, high-end and ultimate platform. As $c_{L}+c_{L L}<c_{U}+c_{U L}<c_{H}+c_{H L}$ and $c_{H}+c_{H H}<c_{U}+c_{U H}<c_{L}+c_{L H}$, both products are derived from their specialized platform $\left(x_{L L}=1\right.$ and $\left.x_{H H}=1\right)$. Hence $\mathcal{C}(L H U)=c_{L} \mu_{L}+c_{H} \mu_{H}+I_{L}+I_{H}+I_{U}$.

13. Platform strategy LMU $\left(x_{L}=1, x_{M}=1\right.$ and $\left.x_{U}=1\right)$ : invest in the development of a low-end, middle and ultimate platform. As $c_{L}+c_{L L}<c_{U}+c_{U L}<c_{M}+c_{M L}$ and $c_{U}+c_{U H}<c_{M}+c_{M H}<c_{L}+c_{L H}$, the low-end product is derived from the low-end platform $\left(x_{L L}=1\right)$ and the high-end product from the ultimate platform $\left(x_{U H}=1\right)$. Hence $\mathcal{C}(L M U)=$ $c_{U H} \mu_{H}+c_{L} \mu_{L}+c_{U} \mu_{H}+I_{L}+I_{M}+I_{U}$.

14. Platform strategy MHU $\left(x_{M}=1, x_{H}=1\right.$ and $\left.x_{U}=1\right)$ : invest in the development of a middle, high-end and ultimate platform. As $c_{U}+c_{U L}<c_{M}+c_{M L}<c_{H}+c_{H L}$ and $c_{H}+c_{H H}<c_{U}+c_{U H}<c_{M}+c_{M H}$, the low-end product is derived from the ultimate platform $\left(x_{U L}=1\right)$ and the high-end product from the high-end platform $\left(x_{H H}=1\right)$. Hence $\mathcal{C}(M H U)=c_{U L} \mu_{L}+c_{U} \mu_{L}+c_{H} \mu_{H}+I_{M}+I_{H}+I_{U}$

15. Platform strategy LMHU $\left(x_{L}=1, x_{M}=1, x_{H}=1\right.$ and $\left.x_{U}=1\right)$ : invest in the development of a low-end, middle, high-end and ultimate platform. As $c_{L}+c_{L L}<c_{U}+c_{U L}<$ $c_{M}+c_{M L}<c_{H}+c_{H L}$ and $c_{H}+c_{H H}<c_{U}+c_{U H}<c_{M}+c_{M H}<c_{L}+c_{L H}$, derive the low-end product from the low-end platform $\left(x_{L L}=1\right)$ and the high-end product from the high-end platform $\left(x_{H H}=1\right)$. Hence $\mathcal{C}(L M H U)=c_{L} \mu_{L}+c_{H} \mu_{H}+I_{L}+I_{M}+I_{H}+I_{U}$. 
First, we show that developing more platforms than products (platform strategies $L M H, L H U$, $L M U, M H U$ and $L M H U$ ) can never be optimal. We show that these platform strategies are always outperformed by another platform strategy.

Proof.

$$
\begin{aligned}
\mathcal{C}(L H) & <\mathcal{C}(L M H) \\
I_{L}+I_{H}+c_{L} \mu_{L}+c_{H} \mu_{H} & <I_{L}+I_{M}+I_{H}+c_{L} \mu_{L}+c_{H} \mu_{H}
\end{aligned}
$$

which is true since $I_{M}>0$.

Proof.

$$
\begin{aligned}
\mathcal{C}(L H) & <\mathcal{C}(L H U) \\
I_{L}+I_{H}+c_{L} \mu_{L}+c_{H} \mu_{H} & <I_{L}+I_{H}+I_{U}+c_{L} \mu_{L}+c_{H} \mu_{H}
\end{aligned}
$$

which is true since $I_{U}>0$.

Proof.

$$
\begin{aligned}
\mathcal{C}(L H) & <\mathcal{C}(L M U) \\
I_{L}+I_{H}+c_{L} \mu_{L}+c_{H} \mu_{H} & <c_{U H} \mu_{H}+I_{L}+I_{M}+I_{U}+c_{U} \mu_{H}+c_{L} \mu_{L} \\
I_{M}+I_{U}-I_{H}+\left(c_{U}+c_{U H}-c_{H}\right) \mu_{H} & >0
\end{aligned}
$$

which is true since $c_{U}>c_{H}, c_{U H}>0, \mu_{H}>0, I_{M}>0$ and $I_{U}>I_{H}$.

Proof.

$$
\begin{aligned}
\mathcal{C}(L H) & <\mathcal{C}(M H U) \\
I_{L}+I_{H}+c_{L} \mu_{L}+c_{H} \mu_{H} & <c_{U} \mu_{L}+I_{M}+I_{H}+I_{U}+c_{U} \mu_{L}+c_{H} \mu_{H} \\
c_{U L} \mu_{L}+I_{M}+I_{U}-I_{L}+\left(c_{U}-c_{L}\right) \mu_{L} & >0
\end{aligned}
$$

which is true since $c_{U L}>0, c_{U}>c_{L}, \mu_{L}>0, I_{M}>0$ and $I_{U}>I_{L}$.

Proof.

$$
\begin{aligned}
\mathcal{C}(L H) & <\mathcal{C}(L M H U) \\
I_{L}+I_{H}+c_{L} \mu_{L}+c_{H} \mu_{H} & <I_{L}+I_{M}+I_{H}+I_{U}+c_{L} \mu_{L}+c_{H} \mu_{H} \\
0 & <I_{M}+I_{U},
\end{aligned}
$$


which is true since $I_{M}>0$ and $I_{U}>0$.

Next, we show that developing an ultimate platform in combination with another platform (platform strategies $L U, M U, H U, L H U, L M U, M H U$ and $L M H U$ ) are never optimal. We have already shown that platform strategies $L H U, L M U, M H U$ and $L M H U$ are always outperformed by another platform strategy. We do the same for platform strategies $L U, M U$ and $H U$ :

Proof.

$$
\begin{aligned}
\mathcal{C}(L H) & <\mathcal{C}(L U) \\
I_{L}+I_{H}+c_{L} \mu_{L}+c_{H} \mu_{H} & <c_{U H} \mu_{H}+I_{L}+I_{U}+c_{L} \mu_{L}+c_{U} \mu_{H} \\
c_{U H} \mu_{H}+I_{U}-I_{H}+\left(c_{U}-c_{H}\right) \mu_{H} & >0,
\end{aligned}
$$

which is true since $c_{U H}>0, \mu_{H}>0, c_{U}>c_{H}$, and $I_{U}>I_{H}$.

Proof.

$$
\begin{aligned}
\mathcal{C}(U) & <\mathcal{C}(M U) \\
c_{U L} \mu_{L}+c_{U H} \mu_{H}+I_{U}+c_{U} \mu_{L}+c_{U} \mu_{H} & <c_{U L} \mu_{L}+c_{U H} \mu_{H}+I_{M}+I_{U}+c_{U} \mu_{L}+c_{U} \mu_{H} \\
0 & <I_{M},
\end{aligned}
$$

which is true since $I_{M}>0$.

Proof.

$$
\begin{aligned}
\mathcal{C}(L H) & <\mathcal{C}(H U) \\
I_{L}+I_{H}+c_{L} \mu_{L}+c_{H} \mu_{H} & <c_{U L} \mu_{L}+I_{H}+I_{U}+c_{H} \mu_{H}+c_{U} \mu_{L} \\
c_{U L} \mu_{L}+I_{U}-I_{L}+\left(c_{U}-c_{L}\right) \mu_{L} & >0
\end{aligned}
$$

which is true since $c_{U L}>0, \mu_{L}>0, c_{U}>c_{L}$, and $I_{U}>I_{L}$.

Finally, we show that developing a high-end platform in combination with a middle platform (platform strategies $M H, L M H, M H U$ and $L M H U$ ) are also never optimal. We have already shown that platform strategies $L M H, M H U$ and $L M H U$ are always outperformed. We show that also platform strategy $M H$ is always outperformed by another platform strategy:

Proof.

$$
\begin{aligned}
\mathcal{C}(L H) & <\mathcal{C}(M H) \\
I_{L}+I_{H}+c_{L} \mu_{L}+c_{H} \mu_{H} & >c_{M L} \mu_{L}+I_{M}+I_{H}+c_{H} \mu_{H}+c_{M} \mu_{L} \\
c_{M L} \mu_{L}+I_{M}-I_{L}+\left(c_{M}-c_{L}\right) \mu_{L} & >0
\end{aligned}
$$


which is true since $c_{M L}>0, c_{M}>c_{L}, \mu_{L}>0$ and $I_{M}>I_{L}$.

\section{B Indifference lines where two platform strategies have the same total cost}

$\begin{array}{rll}y_{L, H} & \mathcal{C}(L)=\mathcal{C}(H) & \mu_{H}=\left[\left(I_{H}-I_{L}\right)+\left(c_{H L}+c_{H}-c_{L}\right) \mu_{L}\right] /\left(c_{L H}+c_{L}-c_{H}\right) \\ y_{L, M} & \mathcal{C}(L)=\mathcal{C}(M) & \mu_{H}=\left[\left(I_{M}-I_{L}\right)+\left(c_{M L}+c_{M}-c_{L}\right) \mu_{L}\right] /\left(c_{L H}-c_{M H}+c_{L}-c_{M}\right) \\ y_{L, U} & \mathcal{C}(L)=\mathcal{C}(U) & \mu_{H}=\left[\left(I_{U}-I_{L}\right)+\left(c_{U L}+c_{U}-c_{L}\right) \mu_{L}\right] /\left(c_{L H}+c_{L}-c_{U H}-c_{U}\right) \\ y_{L, L H} & \mathcal{C}(L)=\mathcal{C}(L H) & \mu_{H}=I_{H} /\left(c_{L H}+c_{L}-c_{H}\right) \\ y_{L, L M} & \mathcal{C}(L)=\mathcal{C}(L M) & \mu_{H}=I_{M} /\left(c_{L H}-c_{M H}+c_{L}-c_{M}\right) \\ y_{M, H} & \mathcal{C}(M)=\mathcal{C}(H) & \mu_{H}=\left[\left(I_{H}-I_{M}\right)+\left(c_{H L}-c_{M L}+c_{H}-c_{M}\right) \mu_{L}\right] /\left(c_{M H}+c_{M}-c_{H}\right) \\ y_{H, U} & \mathcal{C}(H)=\mathcal{C}(U) & \mu_{H}=\left[\left(I_{H}-I_{U}\right)+\left(c_{H L}-c_{U L}+c_{H}-c_{U}\right) \mu_{L}\right] /\left(c_{U H}+c_{U}-c_{H}\right) \\ y_{H, L H} & \mathcal{C}(H)=\mathcal{C}(L H) & \mu_{L}=I_{L} /\left(c_{H}+c_{H}-c_{L}\right) \\ y_{H, L M} & \mathcal{C}(H)=\mathcal{C}(L M) & \mu_{H}=\left[\left(I_{H}-I_{M}-I_{L}\right)+\left(c_{H L}+c_{H}-c_{L}\right) \mu_{L}\right] /\left(c_{M H}+c_{M}-c_{H}\right) \\ y_{M, U} & \mathcal{C}(M)=\mathcal{C}(U) & \mu_{H}=\left[\left(I_{U}-I_{M}\right)+\left(c_{U L}-c_{M L}+c_{U}-c_{M}\right) \mu_{L}\right] /\left(c_{M H}-c_{U H}+c_{M}-c_{U}\right) \\ y_{M, L H} & \mathcal{C}(M)=\mathcal{C}(L H) & \mu_{H}=\left[\left(I_{L}+I_{H}-I_{M}\right)+\left(c_{L}-c_{M L}-c_{M}\right) \mu_{L}\right] /\left(c_{M H}+c_{M}-c_{H}\right) \\ y_{M, L M} & \mathcal{C}(M)=\mathcal{C}(L M) & \mu_{L}=I_{L} /\left(c_{M L}+c_{M}-c_{L}\right) \\ y_{U, L H} & \mathcal{C}(U)=\mathcal{C}(L H) & \mu_{H}=\left[\left(I_{L}+I_{H}-I_{U}\right)+\left(c_{L}-c_{U}-c_{U L}\right) \mu_{L}\right] /\left(c_{U H}+c_{U}-c_{H}\right) \\ y_{U, L M} & \mathcal{C}(U)=\mathcal{C}(L M) & \mu_{H}=\left[\left(I_{U}-I_{L}-I_{M}\right)+\left(c_{U L}-c_{L}+c_{U}\right) \mu_{L}\right] /\left(c_{M H}+c_{M}-c_{U}-c_{U H}\right) \\ y_{L M, L H} & \mathcal{C}(L M)=\mathcal{C}(L H) & \mu_{H}=\left(I_{H}-I_{M}\right) /\left(c_{M H}+c_{M}-c_{H}\right)\end{array}$

\section{Deriving the intersection points for Figure 4 .}

Let us formulate the intersection points defining the different $\Omega_{Z}$-regions for the situation presented in Figure 4, based on the indifference lines. For ease of formulation, we denote the difference between the ex-post variable production and customization cost of platform portfolio $z_{1}$ or $z_{2}$ to derive product $L$ or $H$ as resp. $\delta_{L}^{z_{1}, z_{2}}=c_{L}\left(z_{1}\right)-c_{L}\left(z_{2}\right)$ and $\delta_{H}^{z_{1}, z_{2}}=c_{H}\left(z_{1}\right)-c_{H}\left(z_{2}\right)$ with $z_{1}, z_{2} \in \mathcal{Z}$. Note that $\delta_{L}^{z_{1}, z_{2}}=-\delta_{L}^{z_{2}, z_{1}}$ and $\delta_{H}^{z_{1}, z_{2}}=-\delta_{H}^{z_{2}, z_{1}}$. For instance, if $\mathrm{z}_{1}=L$ and $z_{2}=H$, $\delta_{L}^{z_{1}, z_{2}}=\delta_{L}^{L, H}=c_{L}(L)-c_{L}(H)=c_{L}-\left(c_{H}+c_{H L}\right)$. Then, to determine the existence and span of 
the $\Omega_{Z}$ in Figure 4 it suffices to consider the following intersection points:

$$
\begin{aligned}
& A:\left(\mu_{L}, \mu_{H}\right)=\left(\frac{\delta_{H}^{U, M} I_{H}+\delta_{H}^{M, H} I_{U}+\delta_{H}^{H, U} I_{M}}{\delta_{H}^{M, U} \delta_{L}^{H, U}-\delta_{H}^{U, H} \delta_{L}^{U, M}}, \frac{-\delta_{L}^{U, M} I_{H}-\delta_{L}^{M, H} I_{U}-\delta_{L}^{H, U} I_{M}}{\delta_{H}^{M, U} \delta_{L}^{H, U}-\delta_{H}^{U, H} \delta_{L}^{U, M}}\right) \\
& B:\left(\mu_{L}, \mu_{H}\right)=\left(\frac{I_{L}}{\delta_{L}^{H, L}}, \frac{I_{H}-I_{U}+\frac{\delta_{L}^{H, U} I_{L}}{\delta_{L}^{H, L}}}{\delta_{H}^{U, H}}\right) \\
& C:\left(\mu_{L}, \mu_{H}\right)=\left(\frac{\delta_{H}^{M, H} I_{L}+\delta_{H}^{M, U} I_{H}+\delta_{H}^{H, M} I_{U}+\delta_{H}^{U, H} I_{M}}{\delta_{L}^{U, L} \delta_{H}^{M, H}}, \frac{I_{H}-I_{M}}{\delta_{H}^{M, H}}\right) \\
& D:\left(\mu_{L}, \mu_{H}\right)=\left(\frac{\delta_{H}^{L, U} I_{M}+\delta_{H}^{M, L} I_{U}+\delta_{H}^{L, M} I_{L}}{\delta_{L}^{U, L} \delta_{H}^{L, M}}, \frac{I_{M}}{\delta_{H}^{L, M}}\right) \\
& E:\left(\mu_{L}, \mu_{H}\right)=\left(\frac{\delta_{H}^{M, L} I_{U}+\delta_{H}^{U, M} I_{L}+\delta_{H}^{L, U} I_{M}}{\delta_{H}^{L, M} \delta_{L}^{U, M}-\delta_{H}^{M, U} \delta_{L}^{M, L}}, \frac{-\delta_{L}^{M, L} I_{U}-\delta_{L}^{U, M} I_{L}-\delta_{L}^{L, U} I_{M}}{\delta_{H}^{L, M} \delta_{L}^{U, M}-\delta_{H}^{M, U} \delta_{L}^{M, L}}\right) \\
& F:\left(\mu_{L}, \mu_{H}\right)=\left(0, \frac{I_{H}-I_{M}}{\delta_{H}^{M, H}}\right) \\
& G:\left(\mu_{L}, \mu_{H}\right)=\left(0, \frac{I_{M}-I_{L}}{\delta_{H}^{L, M}}\right)
\end{aligned}
$$

with $\Omega_{U}$ determined by $A$ until $E$ and $\Omega_{M}$ by $A, E, F$ and $G, \Omega_{L}$ by $D, E$ and $G, \Omega_{H}$ by $A, B$ and $F, \Omega_{L M}$ by $D$ and $C$ and $\Omega_{L H}$ by $B$ and $C$.

Hereafter, we illustrate how the expression of intersection point $A$ was obtained. A similar logic is applied to find the other intersection points.

Reconstruction of intersection point A:

In point $\mathrm{A}$ it holds that intersection line $y_{H, U}$ crosses intersection line $y_{M, U}$ so that:

$$
\begin{aligned}
y_{H, U} & =y_{M, U} \\
\mu_{H}=\frac{\left[\left(I_{H}-I_{U}\right)+\left(c_{H L}-c_{U L}+c_{H}-c_{U}\right) \mu_{L}\right]}{\left(c_{U H}+c_{U}-c_{H}\right)} & =\frac{\left[\left(I_{U}-I_{M}\right)+\left(c_{U L}-c_{M L}+c_{U}-c_{M}\right) \mu_{L}\right]}{\left(c_{M H}-c_{U H}+c_{M}-c_{U}\right)} .
\end{aligned}
$$

Using the notation $\delta_{j}^{z_{1}, z_{2}}$, the above expression then simplifies to:

$$
\mu_{H}=\frac{\left(I_{H}-I_{U}\right)+\delta_{L}^{H, U} \mu_{L}}{\delta_{H}^{U, H}}=\frac{\left(I_{U}-I_{M}\right)+\delta_{L}^{U, M} \mu_{L}}{\delta_{H}^{M, U}} .
$$

This means:

$$
\delta_{H}^{M, U} I_{H}-\delta_{H}^{M, U} I_{U}-\delta_{H}^{U, H} I_{U}+\delta_{H}^{U, H} I_{M}=\left(\delta_{H}^{U, H} \delta_{L}^{U, M}-\delta_{H}^{M, U} \delta_{L}^{H, U}\right) \mu_{L} .
$$

As $\delta_{H}^{M, U}+\delta_{H}^{U, H}=c_{H}(M)-c_{H}(U)+c_{H}(U)-c_{H}(H)=c_{H}(M)-c_{H}(H)=\delta_{H}^{M, H}, \mu_{L}$ simplifies to: 


$$
\mu_{L}=\frac{\delta_{H}^{M, U} I_{H}-\delta_{H}^{M, H} I_{U}+\delta_{H}^{U, H} I_{M}}{\delta_{H}^{U, H} \delta_{L}^{U, M}-\delta_{H}^{M, U} \delta_{L}^{H, U}}
$$

As $\delta_{j}^{z_{1}, z_{2}}=-\delta_{j}^{z_{2}, z_{1}}, \mu_{L}$ can be written as:

$$
\mu_{L}=\frac{\delta_{H}^{U, M} I_{H}+\delta_{H}^{M, H} I_{U}+\delta_{H}^{H, U} I_{M}}{\delta_{H}^{M, U} \delta_{L}^{H, U}-\delta_{H}^{U, H} \delta_{L}^{U, M}}
$$

We replace $\mu_{L}$ (given by expression (36) ) in expression (35) to find $\mu_{H}$ :

$$
\begin{aligned}
\mu_{H} & =\frac{\left[\left(I_{H}-I_{U}\right)+\delta_{L}^{H, U} \mu_{L}\right]}{\delta_{H}^{U, H}} \\
& =\frac{\left(\delta_{H}^{M, U} \delta_{L}^{H, U}-\delta_{H}^{U, H} \delta_{L}^{U, M}\right)\left(I_{H}-I_{U}\right)+\delta_{L}^{H, U}\left(\delta_{H}^{U, M} I_{H}+\delta_{H}^{M, H} I_{U}+\delta_{H}^{H, U} I_{M}\right)}{\left(\delta_{H}^{M, U} \delta_{L}^{H, U}-\delta_{H}^{U, H} \delta_{L}^{U, M}\right) \delta_{H}^{U, H}} \\
& =\frac{\left(-\delta_{H}^{U, H} \delta_{L}^{U, M}\right) I_{H}+\left(\delta_{H}^{U, H} \delta_{L}^{U M}+\delta_{L}^{H, U}\left(-\delta_{H}^{M, U}+\delta_{H}^{M, H}\right)\right) I_{U}+\left(\delta_{L}^{H, U} \delta_{H}^{H, U} I_{M}\right)}{\left(\delta_{H}^{M, U} \delta_{L}^{H, U}-\delta_{H}^{U, H} \delta_{L}^{U, M}\right) \delta_{H}^{U, H}} .
\end{aligned}
$$

Since $-\delta_{H}^{M, U}+\delta_{H}^{M, H}=-\left(c_{H}(M)-c_{H}(U)\right)+\left(c_{H}(M)-c_{H}(H)\right)=c_{H}(U)-c_{H}(H)=\delta_{H}^{U, H}, \mu_{H}$ simplifies to:

$$
\begin{gathered}
\mu_{H}=\frac{\left(-\delta_{H}^{U, H} \delta_{L}^{U, M}\right) I_{H}+\left(\delta_{H}^{U, H} \delta_{L}^{U, M}+\delta_{L}^{H, U} \delta_{H}^{U, H}\right) I_{U}+\left(\delta_{L}^{H, U} \delta_{H}^{H, U} I_{M}\right)}{\left(\delta_{H}^{M, U} \delta_{L}^{H, U}-\delta_{H}^{U, H} \delta_{L}^{U, M}\right) \delta_{H}^{U, H}} \\
\mu_{H}=\frac{-\delta_{L}^{U, M} I_{H}+\left(\delta_{L}^{U, M}+\delta_{L}^{H, U}\right) I_{U}-\delta_{L}^{H, U} I_{M}}{\left(\delta_{H}^{M, U} \delta_{L}^{H, U}-\delta_{H}^{U, H} \delta_{L}^{U, M}\right)}
\end{gathered}
$$

As $\delta_{L}^{U, M}+\delta_{L}^{H, U}=c_{L}(U)-c_{L}(M)+c_{L}(H)-c_{L}(U)=c_{L}(H)-c_{L}(M)=\delta_{L}^{H, M}$, and using expression (36) and (37), the coordinates of the intersection point $A$ are given by:

$$
\left(\mu_{L}, \mu_{H}\right)=\left(\frac{\delta_{H}^{U, M} I_{H}+\delta_{H}^{M, H} I_{U}+\delta_{H}^{H, U} I_{M}}{\delta_{H}^{M, U} \delta_{L}^{H, U}-\delta_{H}^{U, H} \delta_{L}^{U, M}}, \frac{-\delta_{L}^{U, M} I_{H}-\delta_{L}^{M, H} I_{U}-\delta_{L}^{H, U} I_{M}}{\delta_{H}^{M, U} \delta_{L}^{H, U}-\delta_{H}^{U, H} \delta_{L}^{U, M}}\right)
$$

A similar reasoning is followed to find the coordinates of the other intersection points. 


\section{Formulation of the threshold investment cost where platform strategy $\mathrm{M}$ and $\mathrm{U}$ in Figure 4 are never optimal}

The coordinates $\left(x_{p}, y_{p}\right)$ are used for the intersection point $p \in\{A, B, C, D, E, F, G\}$ (given earlier in Appendix) and $\left(x_{l_{1} \cap l_{2}}, y_{l_{1} \cap l_{2}}\right)$ are the coordinates where indifference line $l_{1}$ and $l_{2}$ cross. We can identify the maximal or threshold investment cost for the single flexible platforms $M$ and $U$ to be optimal.

Under the Figure 4 scenario, the single flexible platform strategy $M$ cannot be optimal if its investment cost $I_{M} \geq \bar{I}_{M}$, where the threshold cost $\bar{I}_{M}=\min \left\{\bar{I}_{M, 1}, \bar{I}_{M, 2}\right\}$. These threshold costs are found based on the intersection points $A$ to $G$ : we look for the conditions of the intersection points so that the $\Omega_{M}$-region does not exist. Then:

$$
\begin{aligned}
& y_{A} \leq y_{F} \text { and } y_{E} \leq y_{G} \text { (Platform strategy } U \text { outperforms platform strategy } M \text { ) } \\
& \rightarrow \bar{I}_{M, 1}=\max \left\{\frac{\delta_{H}^{U M} I_{H}+\delta_{H}^{M H} I_{U}}{\delta_{H}^{H U}}, \frac{\delta_{H}^{L M} I_{U}+\delta_{H}^{M U} I_{L}}{\delta_{H}^{L U}}\right\} \\
& \left.y_{E} \geq y_{A} \text { and } y_{F} \leq y_{G} \text { (Platform strategy } L H \text { or } L \text { outperform platform strategy } M\right) \\
& \rightarrow \bar{I}_{M, 2}=\max \left\{\frac{\left(\delta_{H}^{M U} \delta_{L}^{H U}-\delta_{H}^{U H} \delta_{L}^{U M}\right)\left(I_{L} \delta_{L}^{U M}+\delta_{L}^{M L} I_{U}\right)+\left(\delta_{H}^{L M} \delta_{L}^{U M}-\delta_{H}^{M U} \delta_{L}^{M L}\right)\left(\delta_{L}^{M U} I_{H}+\delta_{L}^{H M} I_{U}\right)}{\left(\delta_{H}^{L M} \delta_{L}^{U M}-\delta_{H}^{M U} \delta_{L}^{M L}\right) \delta_{L}^{H U}-\delta_{L}^{L U}\left(\delta_{H}^{M U} \delta_{L}^{H U}-\delta_{H}^{U H} \delta_{L}^{U M}\right)}, \frac{\delta_{H}^{L M} I_{H}+\delta_{H}^{M H} I_{L}}{\delta_{H}^{L H}}\right\}
\end{aligned}
$$

When $I_{M} \geq \bar{I}_{M}$, the region $\Omega_{M}$ ceases to exist and a single flexible platform strategy $M$ cannot be optimal. Similar results apply to the single flexible platform strategy $U$ :

Under the Figure 4 scenario, the single flexible platform strategy $U$ cannot be optimal if its investment cost $I_{U} \geq \bar{I}_{U}$, where the threshold cost $\bar{I}_{U}=\min \left\{\bar{I}_{U, 1}, \bar{I}_{U, 2}, \bar{I}_{U, 3}\right\}$. These threshold costs are found based on the intersection points $A$ to $G$ : we look for the conditions of the intersection points so that the $\Omega_{U}$-region does not exist. Then:

$$
\begin{aligned}
& y_{E} \geq y_{A} \text { and } y_{y_{U, L H} \cap y_{L, U}} \geq y_{B} \text { (Platform strategy } L \text { or } H \text { outperforms platform strategy } U \text { ) } \\
& \rightarrow \bar{I}_{U, 1}=\max \left\{\frac{\left(\delta_{H}^{L M} \delta_{L}^{U M}-\delta_{H}^{M U} \delta_{L}^{M L}\right)\left(\delta_{L}^{M U} I_{H}+\delta_{L}^{U H} I_{M}\right)+\left(\delta_{H}^{M U} \delta_{L}^{H U}-\delta_{H}^{U H} \delta_{L}^{U M}\right)\left(\delta_{L}^{U M} I_{L}+\delta_{L}^{L U} I_{M}\right)}{\left(\delta_{H}^{L M} \delta_{L}^{U M}-\delta_{H}^{M U} \delta_{L}^{M L}\right) \delta_{L}^{M H}+\left(\delta_{H}^{M U} \delta_{L}^{H U}-\delta_{H}^{U H} \delta_{L}^{U M}\right) \delta_{L}^{L M}}, \frac{\delta_{H}^{L U} I_{H}}{\delta_{H}^{L H}}+\frac{\delta_{L}^{H U} I_{L}}{\delta_{L}^{H L}}\right\} \\
& y_{C} \geq y_{B} \text { and } y_{y_{U, L M} \cap y_{M, U}} \geq y_{A} \text { (Platform strategy } L M \text { or } H \text { outperforms platform strategy } U \text { ) } \\
& \rightarrow \bar{I}_{U, 2}=\max \left\{\frac{\delta_{H}^{M U} I_{H}+\delta_{H}^{U H} I_{M}}{\delta_{H}^{M H}}+\frac{\delta_{L}^{H U} I_{L}}{\delta_{L}^{H L}}, \frac{\left(\delta_{H}^{M U} \delta_{L}^{H U}-\delta_{H}^{U H} \delta_{L}^{U M}\right) I_{L} \delta_{L}^{U M}+\delta_{L}^{M L}\left(\delta_{L}^{U M} I_{H}+\delta_{L}^{H U} I_{M}\right)}{\delta_{L}^{H M} \delta_{L}^{M L}}\right\} \\
& y_{B} \leq y_{A} \text { and } y_{y_{U, L H} \cap y_{L, U}} \leq y_{E} \text { (Platform strategy } M \text { or } L H \text { outperforms platform strategy } U \text { ) } \\
& \rightarrow \bar{I}_{U, 3}=\max \left\{\frac{\delta_{L}^{H L}\left(\delta_{H}^{M U} I_{H}+\delta_{H}^{U H} I_{M}\right)+\left(\delta_{H}^{M U} \delta_{L}^{H U}-\delta_{H}^{U H} \delta_{L}^{U M}\right) I_{L}}{\delta_{L}^{H L} \delta_{H}^{M H}}, \frac{\left(\delta_{H}^{L M} \delta_{L}^{U M}-\delta_{H}^{M U} \delta_{L}^{M L}\right) I_{H}-\delta_{H}^{L H}\left(\delta_{L}^{M U} I_{L}+\delta_{L}^{U L} I_{M}\right)}{\delta_{H}^{L H} \delta_{L}^{L M}}\right\}
\end{aligned}
$$

